\title{
GLOBAL REGULAR SOLUTION FOR THE EINSTEIN-MAXWELL-BOLTZMANN-SCALAR FIELD SYSTEM IN A BIANCHI TYPE-I SPACE-TIME
}

\author{
Noutchegueme Norbert $^{a}$, Dongo David $^{b}$, Djiofack Francis Etienne ${ }^{c}$ \\ ${ }^{a}$ Department of Mathematics, Faculty of Science, University of Yaounde I, \\ POB: 812 Yaounde Cameroun \\ ${ }^{a}$ nnoutch@yahoo.fr \\ ${ }^{b, c}$ Department of Mathematics, Faculty of Science, University of Dschang, \\ POB: 67 Dschang Cameroun \\ ${ }^{b}$ dongodavid@yahoo.fr \\ francisdjiofack@yahoo.fr
}

\section{ABSTRACT}

We prove an existence and uniqueness of regular solution to the Einstein-Maxwell-Boltzmann-Scalar Field system with pseudo-tensor of pressure and the cosmological constant globaly in time. We clarify the choice of the function spaces and we establish step by step all the essential energy estimations leading to the global existence theorem.

\section{Mathematics Subjet Classification: 83Cxx}

Keywords. pseudo-tensor ; cosmological constant ; Einstein system ; Maxwell system;global existence ; regular.

\section{INTRODUCTION}

The basic equation of general relativity are the Einstein equation coupled to some other partial differential equation describing the matter content of space-time. There are many choices of matter model which are of physical interest. Solving the Einstein equations means determining both the gravitational field, subjet to the Einstein equation, and its sources, subjet to other type of equations. If we consider the case of charged particles, we must take into account the Maxwell equation which are the basic equation of Electromagnetism and determine the electromagnetic field $\mathrm{F}$ created by the fast-moving charged particles in the system. We are interested in this work in global dynamics of magnetized relativistic kinetic matter with cosmological constant in the presence of a massive scalar field and pseudo-tensor of pressure on a Bianchi type I space-time with a locally rotationnal symmetric (L.R.S). We consider the case where the electromagnetic field $F$ is generated, through the Maxwell equation by the Maxwell current defined by the distribution function $f$ of the colliding particles, a charge density $e$, and a future pointing unit vector $u$, tangent at any point to the temporal axis. The particles are statistically desccribed in terms of their distribution function, denoted by $f$, which is a non-negative real valued function of both the position and the momentum of particles and which is subject to the Boltzmann equation.

We then consider the Einstein-Maxwell-Boltzmann system with the cosmological constant and pseudo-tensor of pressure in the presence of a massive scalar field. The source term of the Einstein equation then takes the form $8 \pi\left(T_{\alpha \beta}^{1}+\tau_{\alpha \beta}+T_{\alpha \beta}^{2}+H_{\alpha \beta}\right)$, where $\left(T_{\alpha \beta}^{1}\right)$ is the energy-momentum tensor associated to $f ;\left(\tau_{\alpha \beta}\right)$ is the Maxwell tensor associated to the electromagnetic $\mathrm{F} ;\left(T_{\alpha \beta}^{2}\right)$ is the tensor associated to a massive scalar field $\phi$ and $\left(H_{\alpha \beta}\right)$ is the pseudo-tensor of the pressure.

Many authors obtained a global existence theorem of the Einstein equation coupled to various kind of equations. $\mathrm{N}$. Noutchegueme and D. Dongo obtained in [1] a global existence theorem of the Einstein-Boltzmann system in the Bianchi type I space-time, but the solution was not regular; N. Noutchegueme and R. Ayissi in [5] have obtained the same non-regular solution; N. Noutchegueme and R. Ayissi in [6] the Einstein-Maxwell-Boltzmann equation with the cosmological constant; but they did not take account of the scalar field and the pseudo-tensor of pressure. The originality of the present work is based on the fact that we consider the whole system that will be certainly of a great interest in order to model some natural phenomena and to confirm the actual observation concerning our universe.

The paper is organized as follows. In section 2, we introduce our system on a Bianchi type I space-time; in section 3, we present the functional space and the principal result of the regular Boltzmann equation. In section 4, we study the Einstein equation; in section 5, we prove a local in time existence theorem for our coupled system; in section 6 , we prove that the solution obtained in section 5 in global.

\section{EQUATIONS AND PRELIMINARY RESULTS}

- Unless otherwise specifed, Greek indices, $\alpha, \beta, \gamma, \ldots$, range from 0 to 3 and Latin indices, $i, j, k, \ldots$, from 1 to 3 . We adopt the Einstein summation convention 


$$
a_{\alpha} b^{\alpha}=\sum_{\alpha} a_{\alpha} b^{\alpha}
$$

We consider the collisional evolution of a kind of fast moving massive and charged particules in the time-oriented Bianchi type I space-time with L.R.S, the metric then takes the form

$$
g=-d t^{2}+a^{2}(t) d x^{2}+b^{2}(t)\left(d y^{2}+d z^{2}\right),
$$

Where the metric potentials $a>0, b>0$ are two continuously differentiable unknown functions of time $t$ alone and subjet to the Einstein equations.

- The system reads

$$
\begin{gathered}
R_{\alpha \beta}-\frac{1}{2} R g_{\alpha \beta}+\wedge g_{\alpha \beta}=8 \pi\left(T_{\alpha \beta}^{1}+\tau_{\alpha \beta}+T_{\alpha \beta}^{2}+H_{\alpha \beta}\right) \\
\nabla_{\alpha} F^{\alpha \beta}=4 \pi J^{\beta} \\
\nabla_{\alpha} F_{\beta \gamma}+\nabla_{\beta} F_{\gamma \alpha}+\nabla_{\gamma} F_{\alpha \beta}=0 \\
L_{X} f=Q(f, f)
\end{gathered}
$$

Where: (2) is the Einstein system for the unknown metric tensor $g=\left(g_{\alpha \beta}\right) ; \quad R_{\alpha \beta}$ is the Ricci tensor, contracted of the curvature tensor, $R=g^{\alpha \beta} R_{\alpha \beta}$ is the scalar curvature; $\left(T_{\alpha \beta}^{1}\right)$ is the energy-momentum tensor associated to $f,\left(\tau_{\alpha \beta}\right)$ is the Maxwell tensor associated to the electromagnetic field $F ;\left(T_{\alpha \beta}^{2}\right)$ is the tensor associated to a massive scalar field $\phi$ which is an unknown function of the time t; (3) and (4) are the two sets of Maxwell equations written in covariant form, for the electomagnetic field $F=\left(F^{0 i}, F_{i j}\right)$ which is the unknown. $F$ is a closed antisymmetric 2 -form depending only on the time $t, \quad F^{0 i}$ and $F_{i j}$ are respectively its electric and magnetic parts.

$$
T_{\alpha \beta}^{1}, \tau_{\alpha \beta} \text { and } T_{\alpha \beta}^{2} \text { are defined by: }
$$

$$
\begin{gathered}
T_{\alpha \beta}^{1}=\int_{\mathrm{R}^{3}} \frac{f(t, \bar{p}) p_{\alpha} p_{\beta}|g|^{\frac{1}{2}}}{p^{o}} d \stackrel{-}{p}, \\
\tau_{\alpha \beta}=-\frac{1}{4} g_{\alpha \beta} F^{\lambda \mu} F_{\lambda \mu}+F_{\alpha \lambda} F_{\beta}^{\lambda} \\
T_{\alpha \beta}^{2}=\nabla_{\alpha} \phi \nabla_{\beta} \phi-\frac{1}{2} g_{\alpha \beta}\left[\nabla^{\lambda} \phi \nabla_{\lambda} \phi+m_{0}^{2} \phi^{2}\right]
\end{gathered}
$$

where in (6), (7) as in (3) and (4), $f$ is the distribution function which measures the probability of the presence of particules in the plasma, $\mathrm{m}_{0}>0$ is a given constant called the mass of a unknown scalar field $\phi$. Notice that $\frac{1}{2} m_{0}^{2} \phi^{2}$ represents the potential associated to the scalar field $\phi$ and $\nabla$ stands for the covariant derivative. In (2), $H_{\alpha \beta}$ is the pseudo-tensor of pressure and is defined by:

$$
H_{\alpha \beta}=-\theta_{\alpha \beta} \text {, where } \nabla_{\alpha} \theta^{\alpha \beta}=-u^{\beta}, \quad g^{i j} \theta_{i j}=0
$$

with, $u=\left(u^{\beta}\right)=(1,0,0,0)$ a unit future pointing time-like vector, tangent to the axis at any point. (5) is the Boltzmann equation, where $\mathrm{L}_{X}$ is the Lie derivative of $f$ with respect to the vectors field $X(F)=\left(p^{\alpha}, P^{\alpha}(F)\right)$ and $Q(f, f)$ the collision operator we introduce later.

The massive particules have a rest mass $m>0$, normalized to the unity, we denote by $T\left(\mathrm{R}^{4}\right)$ the tangent bundle of 
$\mathrm{R}^{4}$ with coordinates $\left(x^{\alpha}, p^{\alpha}\right)$, where $p=\left(p^{\beta}\right)=\left(p^{0}, \bar{p}\right)$ stands for the momentum of each particule and $\bar{p}=\left(p^{i}\right), i=1,2,3$. In fact, the charged particules move on the mass hyperbolode $P\left(\mathrm{R}^{4}\right) \subset T\left(\mathrm{R}^{4}\right)$, whose equation is $p_{x}(p): g_{x}(p, p)=g_{\alpha \beta} p^{\alpha} p^{\beta}=-1$ or equivalently, using expression (1) of $g$.

$$
p^{0}=\sqrt{1+a^{2}\left(p^{1}\right)^{2}+b^{2}\left(\left(p^{2}\right)+\left(p^{3}\right)^{2}\right)}
$$

where the choice $p^{0}>0$ symbolizes the fact that, naturally, the particles eject towards the future. Due the fact that we are searching an homogeneous space-time, we then have $f: \mathrm{R}^{4} \subset P\left(\mathrm{R}^{4}\right) \rightarrow \mathrm{R}^{+} ;\left(t^{\prime} \bar{p}\right) \mapsto f(t, \bar{p}), f$ is the principal unknown of the Boltzmann equation. We define a scalar product on $\mathrm{R}^{3}$ by setting for $\bar{p}=\left(p^{i}\right)$ and $\bar{q}=$ $\left(q^{i}\right), \bar{p} \cdot \bar{q}=a^{2} p^{1} q^{1}+b^{2}\left(p^{2} q^{2}+p^{3} q^{3}\right)$, we then have $|\bar{p}|_{g}=\sqrt{a^{2}\left(p^{1}\right)^{2}+b^{2}\left(\left(p^{2}\right)+\left(p^{3}\right)^{2}\right)}$.

In the presence of the electromagnetic field $F$, the trajectoires $s \mapsto\left(x^{\alpha}(s), p^{\alpha}(s)\right)$ of the charged particles are no longer the geodesics of space-time $\left(\mathrm{R}^{4}, g\right)$, but the solutions of the differential system

$$
\frac{d x^{\alpha}}{d s}=p^{\alpha}, \frac{d p^{\alpha}}{d s}=P^{\alpha}, P^{\alpha}=-\Gamma_{\lambda \mu}^{\alpha} p^{\lambda} p^{\mu}+e p^{\beta} F_{\beta}^{\alpha}
$$

where $e=e(t) \geq 0$ denotes the charge density of particles. The charged particles also create a current $J=\left(J^{\beta}\right)$ called the Maxwell current that we take in form

$$
J^{\beta}=\int \frac{1}{p^{0}} p^{\beta} f a b^{2} d \bar{p}-e u^{\beta} .
$$

According to Lichnerowicz and Chernikov, we consider a scheme in which at a given position ( $x^{i}$ ), only two particules collide without destroying each other, the sum of their momenta being preserved

$$
p+q=p^{\prime}+q^{\prime}
$$

where $p, q$ stand for momenta before the shock and $p^{\prime}, q^{\prime}$ the momenta after the shock. The collision operator $\mathrm{Q}$ is then defined, using functions $f$ and $g$ on $\mathrm{R}^{3}$ and the above notations, by $Q=Q^{+}-Q^{-}$where

$$
\begin{aligned}
Q^{+}(f, g) & =\int_{\mathrm{R}^{3}} \omega_{q} \int_{S^{2}} f\left(\bar{p}^{\prime}\right) g\left(\bar{q}^{\prime}\right) B\left(a, b, \bar{p}, \bar{q}, \overline{p^{\prime}}, \overline{q^{\prime}}\right) d \omega \\
Q^{-}(f, g) & =\int_{\mathrm{R}^{3}} \omega_{q} \int_{S^{2}} f(\bar{p}) g(\bar{q}) B\left(a, b, \bar{p}, \bar{q}, \bar{p}, \overline{q^{\prime}}\right) d \omega .
\end{aligned}
$$

In which $\omega_{q}=\frac{|\operatorname{det} g|^{\frac{1}{2}} d \bar{q}}{q^{o}}$ and $B$ is a non-negative continuous real valued function of all its arguments, called the collision kernel or the cross-section of the collisions, on which we require the boundedness and Lipschitz continuity assumptions as in [2]. (12) expresses, using (9), the conservation of the quantity

$$
E_{n}=\sqrt{1+|\bar{p}|_{g}^{2}}+\sqrt{1+|\bar{q}|_{g}^{2}}
$$

called the elementary energy of the unit rest mass particles. Since $f=f(t, \bar{p})$, using (9) and (10), the Boltzmann equation (5) takes the form 


$$
\frac{\partial f}{\partial t}+\frac{P^{i}}{p^{0}} \frac{\partial f}{\partial p^{i}}=\frac{1}{p^{0}} Q(f, f)
$$

Next, let us introduce the subgroup $\Delta$ of $\Theta_{3}$ defined, as in [3] by: $N_{\varepsilon, \theta}=\left(\begin{array}{lll}\varepsilon & 0 & 0 \\ 0 & \cos \theta & -\sin \theta \\ 0 & \sin \theta & \cos \theta\end{array}\right), \quad \varepsilon= \pm 1, \theta \in \mathrm{R}$. we require that the initial datum $f_{0}=f(0 ; \bar{p})$ verify

$$
f_{0}(t, N \bar{p})=f_{0}(t, \bar{p}), \quad \forall N \in \Delta
$$

It is proved in [3] that if $f_{0}$ is invariant under $\Delta$ then so will be the solution $f$ of the Boltzmann equation satisfying $f(0, \bar{p})=f_{0}(\bar{p})$.

Now the well-known identity $\nabla_{\alpha} \nabla_{\beta} F^{\alpha \beta}=0$ imposes, given (4) that

$$
\nabla_{\beta} J^{\beta}=0
$$

So (3) also implies that

$$
J^{0}=0 .
$$

As a consequence (see $[4]$, we have

$$
J^{i}=\int_{\mathrm{R}^{3}} \frac{1}{p^{0}} p^{i} f a b^{2} d \bar{p}-e u^{i}=0, i=1,2,3 ;
$$

By (18), expression (11) of $J^{\beta}$ in which we set $\beta=0$ then allows to compute e and gives

$$
e(t)=\int_{\mathrm{R}^{3}} \frac{1}{p^{0}} f a b^{2} d \bar{p},
$$

which shows that $\mathrm{a}, \mathrm{b}$ and $\mathrm{f}$ determine $e$.

Now, using all what precedes, one has

$$
F^{0 i}=\frac{a_{0} b_{0}^{2}}{a b^{2}} E^{i}, F_{i j}=F_{i j}(0)=\varphi_{i j}, F^{0 i}(0)=E^{i} \quad i, j=1,2,3
$$

Next, to derive the equation for the scalar field $\phi$, we use the conservation laws:

$$
\nabla_{\alpha}\left(T^{1, \alpha \beta}+\tau^{\alpha \beta}+T^{2, \alpha \beta}+H^{\alpha \beta}\right)=0
$$

A direct calculation using (6), (7), (8), and the relation $\nabla_{\alpha} T^{1, \alpha \beta}=0$ due to J.EHLERS, gives:

$$
\nabla_{\alpha} \tau^{\alpha \beta}=F_{\lambda}^{\beta} \nabla_{\alpha} F^{\alpha \lambda}, \nabla_{\alpha} H^{\alpha \beta}=u^{\beta}, \nabla_{\alpha} T^{2, \alpha \beta}=\nabla^{\beta} \phi\left(^{*}{ }_{g} \phi-m_{0}^{2} \phi\right)
$$

where ${ }^{*}{ }_{g}=\nabla_{\alpha} \nabla^{\alpha}$ is the d'Alembertian or the wave operator. (21) and (22) give, using the Maxwell equation (3):

$$
\nabla^{\beta} \phi\left(_{g}^{*} \phi-m_{0}^{2} \phi\right)+4 \pi F_{\lambda}^{\beta} J^{\lambda}+u^{\beta}=0
$$

(23) reduces, using (18), (19) and since $\nabla^{i} \phi=0$ to:

$$
\nabla^{0} \phi\left(\nabla_{\alpha} \nabla^{\alpha} \phi-m_{0}^{2} \phi\right)+u^{0}=0
$$


Next, it is easily seen, that: $\nabla_{\alpha} \nabla^{\alpha} \phi=-\ddot{\phi}-3 H \dot{\phi}$ and $\nabla^{0} \phi=g^{00} \nabla_{0} \phi=-\dot{\phi}$. (24) gives then:

$$
\dot{\phi}\left(\ddot{\phi}+3 H \dot{\phi}+m_{0}^{2} \phi\right)+1=0
$$

where

$$
H=-\frac{g^{i j} k_{i j}}{3}, \text { with } k_{i j}=-\frac{1}{2} \partial_{t} g_{i j}
$$

$\mathrm{H}$ is called the Hubble variable. To study this non-linear second order equation in $\phi$, we set $\psi=\frac{1}{2}(\dot{\phi})^{2}$, we choose to look for a non-decreasing and non constant scalar field $\phi$, which means $\dot{\phi}>0$;

$$
\dot{\phi}=\sqrt{2 \psi}
$$

For $\psi_{0} \in \mathrm{R}$, there exists $T>0$ such that $\forall t \in[0, T]$,

$$
\psi(t) \geq \frac{1}{2} \psi_{0} .
$$

A direct calculation shows that the components of the tensor,

$$
\begin{gathered}
T_{\alpha \beta}^{1}, T_{\alpha \beta}^{2}, H_{\alpha \beta}, \tau_{\alpha \beta} \text { defined by (6), (6'),(7) and (8) are given, by } \\
T_{00}^{1}=\int_{\mathrm{R}^{3}} f(t, \bar{p}) p^{0}|g|^{\frac{1}{2}} d \bar{p}, T_{11}^{1}=\int_{\mathrm{R}^{3}} \frac{f(t, \bar{p})\left(g_{11}\right)^{2}\left(p^{1}\right)^{2}|g|^{\frac{1}{2}}}{p^{0}} d \bar{p} \\
T_{22}^{1}=T_{33}^{1}=\int_{\mathrm{R}^{3}} \frac{f(t, \bar{p})\left(g_{22}\right)^{2}\left(p^{2}\right)^{2}|g|^{\frac{1}{2}}}{p^{0}} d \bar{p}, \quad T_{i j}^{1}=0, \quad i \neq j \\
T_{00}^{2}=\frac{1}{2}(\dot{\phi})^{2}+\frac{1}{2} m_{0}^{2} \phi^{2}, T_{0 i}^{2}=0, T_{i j}^{2}=\frac{1}{2} g_{i j}\left(2 \psi-m_{0}^{2} \phi^{2}\right), i, j=1,2,3 \\
H_{00}=-\frac{C}{a b^{2}}+\int_{t_{0}}^{t_{0}+T} a(t) b(t) d t, C \in \mathrm{R}_{-}, H_{0 i}=0, H_{i j}=0, i, j=1,2,3 .
\end{gathered}
$$




$$
\left\{\begin{array}{c}
\tau_{00}=\frac{1}{2}\left[\begin{array}{c}
\left.\left(a_{0} E^{1}\right)^{2}\left(\frac{b_{0}}{b}\right)^{4}+\left(b_{0} E^{2}\right)^{2}\left(\frac{b_{0}^{2}}{a b}\right)^{2}+\left(a_{0} E^{3}\right)^{2}\left(\frac{b_{0}^{2}}{a b}\right)^{2}\right] \\
+\left[\left(\frac{\varphi_{12}}{a b}\right)^{2}+\left(\frac{\varphi_{13}}{a b}\right)^{2}+\left(\frac{\varphi_{23}}{b^{2}}\right)^{2}\right] \\
\tau_{11}=\frac{1}{2}\left[-\left(a a_{0} E^{1}\right)^{2}\left(\frac{b_{0}}{b}\right)^{4}+\left(a_{0}^{2} b_{0}^{2} E^{2}\right)^{2}\left(\frac{b_{0}}{b}\right)^{2}+\left(b_{0}^{2} E^{3}\right)^{2}\left(\frac{b_{0}}{b}\right)^{2}\right] \\
+\frac{1}{2}\left[\left(\frac{\varphi_{12}}{b}\right)^{2}+\left(\frac{\varphi_{13}}{b}\right)^{2}-\left(\frac{a \varphi_{23}}{b^{2}}\right)^{2}\right] \\
+\frac{1}{2}\left[\left(\frac{\varphi_{12}}{a}\right)^{2}-\left(\frac{\varphi_{13}}{a}\right)^{2}+\left(\frac{a \varphi_{23}}{b}\right)^{2}\right] \\
\tau_{22}=\frac{1}{2}\left[\left(a_{0} b_{0} E^{1}\right)^{2}\left(\frac{b_{0}}{b}\right)^{2}-\left(b_{0}^{2} E^{2}\right)^{2}\left(\frac{a_{0}}{a}\right)^{2}+\left(b_{0}^{2} E^{3}\right)^{2}\left(\frac{a_{0}}{a}\right)^{2}\right] \\
\tau_{33}=\frac{1}{2}\left[\left(a_{0} b_{0} E^{1}\right)^{2}\left(\frac{b_{0}}{b}\right)^{2}+\left(b_{0}^{2} E^{2}\right)^{2}\left(\frac{a_{0}}{a}\right)^{2}-\left(b_{0}^{2} E^{3}\right)^{2}\left(\frac{a_{0}}{a}\right)^{2}\right] \\
+\frac{1}{2}\left[-\left(\frac{\varphi_{12}}{a}\right)^{2}+\left(\frac{\varphi_{13}}{a}\right)^{2}+\left(\frac{\varphi_{23}}{b}\right)^{2}\right] \\
\tau_{13}=\frac{-1}{b^{2}}\left(a_{0}^{2} b_{0}^{4} E^{1} E^{3}+\varphi_{12} \varphi_{23}\right), \tau_{23}=\frac{1}{a^{2}}\left(-a_{0}^{2} b_{0}^{4} E^{2} E^{3}+\varphi_{12} \varphi_{13}\right) \\
-\left(\frac{a_{0}}{a}\right)\left(\frac{b_{0}}{b}\right)^{2} E^{j} \varphi_{i j}, \quad \tau_{12}=\frac{1}{b^{2}}\left(-a_{0}^{2} b_{0}^{2} E^{1} E^{2}+\varphi_{13} \varphi_{23}\right)
\end{array}\right.
\end{array}\right.
$$

Proposition 1: The system can be written in the form:

$$
\begin{aligned}
& 2 \frac{\dot{a} \dot{b}}{a b}+\left(\frac{\dot{b}}{b}\right)^{2}-\wedge=8 \pi\left[T_{00}^{1}+\tau_{00}+T_{00}^{2}+H_{00}\right] \\
& -a^{2}\left[2 \frac{\ddot{b}}{b}+\left(\frac{\dot{b}}{b}\right)^{2}-\wedge\right]=8 \pi\left[T_{11}^{1}+\tau_{11}+T_{11}^{2}\right] \\
& -b^{2}\left[\frac{\ddot{b}}{b}+\frac{\ddot{a}}{a}+\frac{\ddot{a} \dot{b}}{a b}-\wedge\right]=8 \pi\left[T_{22}^{1}+\tau_{22}+T_{22}^{2}\right] \\
& F^{0 i}=\frac{a_{0} b_{0}^{2}}{a b^{2}} F^{0 i}(0), F_{i j}=F_{i j}(0)=\varphi_{i j}, \quad i=1,2,3 \\
& \frac{\partial f}{\partial t}+\left(-2 \Gamma_{0 j}^{i} p^{j}+\left(-a_{0} b_{0}^{2} E^{i}+\frac{a b^{2} g^{i i} p^{k} \varphi_{k i}}{p^{0}}\right) \int_{\mathrm{R}^{3}} f d \bar{p}\right) \frac{\partial f}{\partial p^{i}}=\frac{Q(f, f)}{p^{0}}
\end{aligned}
$$




$$
\begin{gathered}
\dot{\phi}=\sqrt{2 \psi} \\
\dot{\psi}=-6 H \psi-m_{0}^{2} \phi \sqrt{2 \psi}-1
\end{gathered}
$$

with: $T_{22}^{1}=T_{33}^{1}, T_{22}^{2}+\tau_{22}=T_{33}^{2}+\tau_{33}, T_{\alpha \beta}^{1}+\tau_{\alpha \beta}+T_{\alpha \beta}^{2}+H_{\alpha \beta}=0$ for $\alpha \neq \beta$.

proof: Simply write Einstein equation (2) for $\alpha=\beta=0$ to obtain (33), for $\alpha=\beta=i$ to obtain (34) and (35). (28) in (27) to obtain (39). Now for $\alpha \neq \beta: T_{\alpha \beta}^{1}+\tau_{\alpha \beta}+T_{\alpha \beta}^{2}+H_{\alpha \beta}=0$, we add the problem of constraints $T_{\alpha \beta}^{1}+\tau_{\alpha \beta}+T_{\alpha \beta}^{2}+H_{\alpha \beta}=0$ if $\alpha \neq \beta$ and $T_{22}^{1}+\tau_{22}+T_{22}^{2}+H_{22}=T_{33}^{1}+\tau_{33}+T_{33}^{2}+H_{33}$, since the Einstein tensor $\mathrm{S}_{\alpha \beta}=0$ if $\alpha \neq \beta ; \mathrm{S}_{22}=S_{33}$, it is proved in [2] that $T_{\alpha \beta}^{1}=0$ if $\alpha \neq \beta, T_{22}^{1}=T_{33}^{1}$, since $H_{i j}=0$, so $\tau_{\alpha \beta}+T_{\alpha \beta}^{2}=0$ if $\alpha \neq \beta$ and $\tau_{22}+T_{22}^{2}=\tau_{33}+T_{33}^{2}+$

\section{- The constraint equations}

Lemma 1: $1^{\circ}$ ) Einstein equation (33), called the Hamiltonian constraint, is satisfied all over the domain of the solutions $a$ and $b$ of (34)-(35), if and only if, the initial data

$a_{0}, \dot{a}_{0}, b_{0}, \dot{b}_{0}, f_{0}, E^{i}, \phi_{0}, \psi_{0}, \varphi_{i j}$ satisfy the condition:

$$
2 \frac{a_{0} \dot{b}_{0}}{a_{0} b_{0}}+\left(\frac{\dot{b}_{0}}{b_{0}}\right)^{2}=\Lambda+\rho_{0}
$$

where

$$
\begin{aligned}
& \rho_{0}=8 \pi \int_{\mathrm{R}^{3}} f_{0}(\bar{p}) \sqrt{1+a_{0}^{2}\left(p^{1}\right)^{2}+b_{0}^{2}\left[\left(p^{2}\right)^{2}+\left(p^{3}\right)^{2}\right]} a_{0} b_{0}^{2} d \bar{p} \\
& +\frac{1}{2}\left[\left(a_{0} E_{0}^{1}\right)^{2}+\left(b_{0} E_{0}^{2}\right)^{2}+\left(b_{0} E_{0}^{3}\right)^{2}\right] \\
& +4 \pi\left[\left(\frac{\varphi_{12}}{a_{0} b_{0}}\right)^{2}+\left(\frac{\varphi_{13}}{a_{0} b_{0}}\right)^{2}+\left(\frac{\varphi_{23}}{b_{0}^{2}}\right)^{2}\right]+8 \pi\left[\psi_{0}+\frac{1}{2} m_{0}^{2} \phi_{0}^{2}\right] \\
& +8 \pi\left[-\frac{C}{a_{0}^{2} b_{0}}+\int_{0}^{T} a_{0}(t) b_{0}(t) d t\right], C \leq 0 .
\end{aligned}
$$

$2^{\circ}$ ) The remaining Einstein equations

$$
S_{i}^{o}+\Lambda g_{i}^{0}=8 \pi\left(T_{i}^{1,0}+\tau_{i}^{0}+T_{i}^{2,0}+H_{i}^{0}\right), S_{i j}+\Lambda g_{i j}=8 \pi\left(T_{i j}^{1}+\tau_{i j}+T_{i j}^{2}+H_{i j}\right) .
$$

are identically satisfied by any solutions a and $\mathrm{b}$ of (33)-(35) if the initiale data $a_{0}, b_{0}, E^{i}, \varphi_{i j}$, verify

$$
\begin{gathered}
E^{i} \varphi_{i j}=0 \\
\sum_{k=1}^{3} \varphi_{i k} \varphi_{j k}-a_{0}^{2} b_{0}^{4} E^{i} E^{j}=0, i \neq j \\
\varphi_{12}^{2}-\varphi_{13}^{2}-a_{0}^{2} b_{0}^{4}\left[\left(E^{2}\right)^{2}-\left(E^{3}\right)^{2}\right]=0
\end{gathered}
$$




\section{proof: See [6].}

Remark 1: In what follows, we suppose that the initial data $a_{0}, \dot{a}_{0}, b_{0}, \dot{b}_{0}, f_{0}, E^{i}, \phi_{0}, \psi_{0}, \varphi_{i j}$ verify the constraint (40) and (42)-(44). One must also remark that if the cosmological constant $\Lambda$ is positive and if $a_{0}, b_{0}$, $f_{0}, E^{i}, \phi_{0}, \psi_{0}, \varphi_{i j}$ are given, then it suffices to deduce $\dot{a}_{0}>0$ and $\dot{b}_{0}>0$.

\section{FUNCTION SPACES AND LOCAL SOLUTION OF THE BOLTZMANN EQUATION}

We define now the function spaces in which we are searching the solution of the system, We also state some useful energy estimations.

Définition 1: Let $T>0, l \in \mathrm{N}$ and $d \in \mathrm{R}$ be given. We define

a) $H_{d}^{l}\left(\mathrm{R}^{3}\right)=\left\{u: \mathrm{R}^{3} \rightarrow \mathrm{R},(1+|\bar{p}|)^{d+|\beta|} \partial \frac{\beta}{p} u \in L^{2}\left(\mathrm{R}^{3}\right),|\beta| \leq l\right\}, H_{d}^{l}\left(\mathrm{R}^{3}\right)$ will be endowed with the norm $\|u\|_{H_{d}^{l}\left(\mathrm{R}^{3}\right)}=\max _{0 \leq \beta \leq l}\left\|(1+|\bar{p}|)^{d+|\beta|} \partial_{\frac{\beta}{p}} u\right\|_{L^{2}\left(\mathrm{R}^{3}\right)}$.

b) $H_{d}^{l}\left(0, T, \mathrm{R}_{\bar{p}}^{3}\right)=\left\{\begin{array}{c}u \in C\left([0, T], C\left(\mathrm{R}_{\bar{p}}^{3}\right)\right), \\ (1+|\bar{p}|)^{d+|\beta|} \partial \frac{\beta}{p} u(t, .) \in L^{2}\left(\mathrm{R}^{3}\right),|\beta| \leq l, \quad 0 \leq t \leq T\end{array}\right\}$

The norm we consider for

$H_{d}^{l}\left(0, T, \mathrm{R}_{\bar{p}}^{3}\right)$ is $\|u\|_{H_{d}^{l}\left(0, T, \mathrm{R}^{\left.\frac{3}{p}\right)}\right.}=\max _{0 \leq \beta \leq l} \sup _{0 \leq t \leq T}\left\|(1+|\bar{p}|)^{d+|\beta|} \partial \frac{\beta}{p} u(t, .)\right\|_{L^{2}\left(\mathrm{R}_{\bar{P}}\right)}$.

c) $H_{d, r}^{l}\left(0, T, \mathrm{R}_{\bar{p}}^{3}\right)=\left\{u \in H_{d}^{l}\left(0, T, \mathrm{R}_{\bar{p}}^{3}\right),\|u\|_{H_{d}^{l}\left(0, T, \mathrm{R}_{p}\right)} \leq r\right\}$, for $r>0$.

Endowed with the induced distance by the norm \|\|$_{H_{d, r}^{l}\left(0, T, R \frac{3}{p}\right)}$ is a complete metric subspace of $H_{d}^{l}\left(0, T, \mathrm{R}_{p}^{3}\right)$.

Remark 2: We choose as in $[2], l=3$ and $\mathrm{d}>\frac{5}{2}$ and we then have $H_{d}^{3}\left(\mathrm{R}^{3}\right) H^{3}\left(\mathrm{R}^{3}\right)^{\rho} \mathrm{C}_{b}^{1}\left(\mathrm{R}^{3}\right)$.

Proposition 2: Let $d \in] \frac{5}{2},+\infty\left[\right.$ be a real number, if $f_{1,} f_{2} \in H_{d}^{3}\left(\mathrm{R}^{3}\right)$, then $\frac{1}{p^{0}} Q\left(f_{1}, f_{2}\right) \in H_{d}^{3}\left(\mathrm{R}^{3}\right)$ and we have $C=C(T)>0 \quad$ such that

$$
\left\|\frac{1}{p^{0}} Q\left(f_{1}, f_{2}\right)\right\|_{H_{d}^{3}\left(\mathrm{R}^{3}\right)} \leq C\left\|f_{1}\right\|_{H_{d}^{3}\left(\mathrm{R}^{3}\right)}\left\|f_{2}\right\|_{H_{d}^{3}\left(\mathrm{R}^{3}\right)}
$$

Moreover

$$
\left\|\frac{1}{p^{0}} Q\left(f_{1}, f_{1}\right)-\frac{1}{p^{0}} Q\left(f_{2,} f_{2}\right)\right\|_{H_{d}^{3}\left(\mathrm{R}^{3}\right)} \leq 2 C\left(\left\|f_{1}\right\|_{H_{d}^{3}\left(\mathrm{R}^{3}\right)}+\left\|f_{2}\right\|_{H_{d}^{3}\left(\mathrm{R}^{3}\right)}\right)\left\|f_{1}-f_{2}\right\|_{H_{d}^{3}\left(\mathrm{R}^{3}\right)} .
$$

proof: see [2] 
Theorem 1: Let $a_{0}, \dot{a}_{0}, b_{0}, \dot{b}_{0}, f_{0}, E^{i}, \phi_{0}, \psi_{0}, \varphi_{i j} \quad$ satistify the conditions (40), (42)-(44). Let $(\square, \vec{a}, \vec{b})$ be fixed such that $\left|\frac{\dot{a}}{a}\right| \leq C_{0}$ and $\left|\frac{\dot{b}}{b}\right| \leq C_{0}, C_{0}$ a constant . Then the Boltzmann equation

$$
\frac{\partial f}{\partial t}+\left[-2 \Gamma_{0 j}^{i} p^{j}+\left(-a_{0} b_{0}^{2} E^{i}+\frac{\square \bar{b}^{2} \stackrel{\square}{g}^{i j} p^{k} \varphi_{k i}}{p^{0}}\right) \int_{\mathrm{R}^{3}} f(t, \bar{p}) d \bar{p}\right] \frac{\partial f}{\partial p^{i}}=\frac{1}{p^{0}} Q(f, f)
$$

has in $H_{d}^{3}\left(0, T, \mathrm{R}_{\frac{1}{p}}^{3}\right)$ a local unique and bounded * -weakly solution $f$ such that $f(0)=f_{0}$.

proof: similar to the one in [2].

\section{LOCAL EXISTENCE OF SOLUTION TO EINSTEIN SYSTEM}

We consider the Einstein system (33)-(35) and the sources terms as

$$
\left\{\begin{array}{c}
\rho=8 \pi\left[T_{00}^{1}+\tau_{00}+T_{00}^{2}+H_{00}\right], P_{1}=\frac{1}{a^{2}} 8 \pi\left[T_{11}^{1}+\tau_{11}+T_{11}^{2}\right] \\
P_{2}=\frac{1}{b^{2}} 8 \pi\left[T_{22}^{1}+\tau_{22}+T_{22}^{2}\right], \square=\frac{P_{1}+2 P_{2}}{\rho}, \stackrel{\square}{R}=\frac{P_{2}-P_{1}}{\rho}
\end{array}\right.
$$

Next, following N.Noutchegueme and D.Dongo in [1], we make the change of variables as indicated below

$$
H=-\frac{t r k}{3}, z=\frac{a^{2} b^{2}}{2 a^{2}+b^{2}+a^{2} b^{2}}, s=\frac{b^{2}}{a^{2}+2 b^{2}}, \Sigma_{+}=-\frac{3}{\operatorname{trk}} \frac{\dot{b}}{b}-1, \operatorname{trk}=-\left(\frac{\dot{a}}{a}+2 \frac{\dot{b}}{b}\right) .
$$

we also set

$$
\bar{\Omega}=\frac{\rho}{3 H^{2}}, \quad q=2 \Sigma_{+}+\frac{\square}{2}(1+\stackrel{\square}{R}), \Sigma_{+} \in[-1,1]
$$

$\mathrm{q}$ is the deceleration parameter and $\Omega$ is the normalized energy density, which can be written see [6]

$$
\stackrel{\square}{\Omega}=1-\Sigma_{+}-\frac{\Lambda}{3 H^{2}} .
$$

Using the new notations, we have the folowing immediate consequences of the above definitions, $i=1,2$ :

$$
\begin{aligned}
& s \in] 0,1[, z \in] 0,1\left[, a=\sqrt{\frac{z}{s(1-z)}}, b=\sqrt{\frac{2 z}{(1-s)(1-z)}},\right.
\end{aligned}
$$

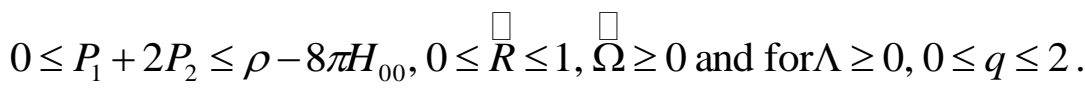

$$
\begin{aligned}
& p^{o}(s, z)=\frac{1}{\sqrt{s(1-s)(1-z)}} \sqrt{s(1-s)(1-z)+z\left[(1-s)\left(p^{1}\right)^{2}+2 s\left(\left(p^{2}\right)^{2}+\left(p^{3}\right)^{2}\right)\right]}
\end{aligned}
$$




$$
\begin{aligned}
& P_{i}(s, z, f, \phi, \psi)=\frac{16 i \pi z^{\frac{5}{2}}}{s^{\left(\frac{5}{2}-i\right)(1-s)^{i}(1-z)^{2}}} \int_{\mathrm{R}^{3}} \frac{f(t, \bar{p})\left(p^{i}\right)^{2}}{p^{0}(s, z)} d \bar{p} \\
& +\frac{\pi(1-s)^{2}(1-z)^{2}}{z^{2}}(-1)^{i}\left(\left(a_{0} b_{0}^{2} E^{1}\right)^{2}+\varphi_{23}^{2}\right) \\
& +\frac{2 \pi s(1-s)(1-z)^{2}}{z^{2}}\left((-1)^{i+1}\left(a_{0} b_{0}^{2} E^{2}\right)^{2}+\left(a_{0} b_{0}^{2} E^{3}\right)^{2}+\varphi_{12}^{2}+(-1)^{i+1} \varphi_{13}^{2}\right)+ \\
& \frac{3}{2}\left(2 \psi-m_{0}^{2} \phi^{2}\right)
\end{aligned}
$$

Proposition 3. Let $\Lambda \geq 0$ and $\dot{b}_{0}<0$, then Einstein systems (33)-(35) have no global solution on $[0,+\infty[$.

Proof. see [6].

Now the system (33)-(35), using the values (51) and(52) can be combined, see $[1]$, to give:

$$
\begin{gathered}
\frac{\ddot{a}}{a}=\frac{2}{3}\left[\left(\frac{\dot{b}}{b}\right)^{2}-\frac{\dot{a} b}{a b}\right]-\frac{\rho}{6}+\frac{1}{2}\left(P_{1}-2 P_{2}\right)+\frac{\Lambda}{3} \\
\frac{\ddot{b}}{b}=\frac{1}{3}\left[\frac{\dot{a} \dot{b}}{a b}-\left(\frac{\dot{b}}{b}\right)^{2}\right]-\frac{\rho}{6}-\frac{1}{2} P_{1}+\frac{\Lambda}{3} .
\end{gathered}
$$

Also notice that

$$
\frac{\dot{a}}{a}=H\left(1-2 \Sigma_{+}\right), \quad \frac{\dot{b}}{b}=H\left(1+\Sigma_{+}\right)
$$

Proposition 4: The Einstein system of equation (53) and (54) can be written as a system of first order in $H, s, z, \Sigma_{+}$ as follows:

$$
\begin{gathered}
\frac{d H}{d t}=-\frac{3}{2}\left(1+\Sigma_{+}^{2}\right) H^{2}-\frac{P_{1}+2 P_{2}}{6}+\frac{\Lambda}{2} \\
\frac{d s}{d t}=6 s(1-s) \Sigma_{+} H \\
\frac{d z}{d t}=2 z(1-z)\left(1+\Sigma_{+}-3 s \Sigma_{+}\right) H \\
\frac{d \Sigma_{+}}{d t}=-\frac{3}{2}\left(1-\Sigma_{+}^{2}\right) H \Sigma_{+}+\frac{P_{1}}{6 H}\left(\Sigma_{+}-2\right)+\frac{P_{2}}{3 H}\left(\Sigma_{+}+1\right)-\frac{\Lambda \Sigma_{+}}{2 H}
\end{gathered}
$$

proof: a) We prove that system (53) and (54) implie system (56)-(59), just by derivating (47), using (53), (54), (55), (48) and (48').

b) Conversely, we prove that (56)-(59) implie (53)-(54). Let $\left(H, s, z, \Sigma_{+}\right)$be a solution of system (56)-(59), we have, 
derivating $\frac{\dot{a}}{a}$ and (55), $\frac{d}{d t}\left(\frac{\dot{a}}{a}\right)=\frac{\ddot{a}}{a}-\left(\frac{\dot{a}}{a}\right)^{2}$ and $\frac{d}{d t}\left(\frac{\dot{a}}{a}\right)=\dot{H}\left(1-2 \Sigma_{+}\right)-2 H \dot{\Sigma}_{+}$before to express (55) then $\frac{\ddot{a}}{a}=\dot{H}\left(1-2 \Sigma_{+}\right)-2 H \dot{\Sigma}_{+}+\left(\frac{\dot{a}}{a}\right)^{2}$. Now, using (56) and (59), and the fact that, by direct compulation

$$
\begin{gathered}
1+q=2 \frac{1}{H^{2}}\left(\frac{\dot{b}}{b}\right)^{2}-4 \frac{\dot{b}}{b H}+3+\frac{1}{6 H^{2}}\left(\rho+P_{1}+2 P_{2}\right) \\
2-q=-2 \frac{1}{H^{2}}\left(\frac{\dot{b}}{b}\right)^{2}+4 \frac{\dot{b}}{b H}-\frac{1}{6 H^{2}}\left(\rho+P_{1}+2 P_{2}\right),
\end{gathered}
$$

one obtains:

$$
\frac{\ddot{a}}{a}==-2\left(\frac{\dot{b}}{b}\right)^{2}+4 \frac{\dot{b}}{b} H-3 H^{2}-\frac{\rho}{6}-\frac{1}{6}\left(P_{1}+2 P_{2}\right)+6 H^{2} \Sigma_{+}+\left(\frac{\dot{a}}{a}\right)^{2}-\frac{2}{3}\left(P_{2}-P_{1}\right)+\frac{\Lambda}{3} ;
$$

Now, using (47), we obtain by direct computation (53). The expression (54) can be obtained by a similar computation. +

Lemma 2: the Hubble variable satisfies the following condition:

$$
\frac{d H}{d t}=-\frac{1}{3}\left(3 H^{2}+L_{i j} L^{i j}-\Lambda+4 \pi g^{i j}\left(T_{i j}^{1}+T_{i j}^{2}\right)+4 \pi\left(T_{00}^{1}+T_{00}^{2}+H_{00}\right)+8 \pi \tau_{00}\right)
$$

where $L_{i j}=k_{i j}+g_{i j} H, k_{i j}$ defined by (26).

proof: We have $H=-\frac{1}{3} g^{i j} k_{i j}$, which implies

$$
\begin{aligned}
& \frac{d H}{d t}=-\frac{1}{3}\left(k_{i j} \frac{d g^{i j}}{d t}+g^{i j} \frac{d k_{i j}}{d t}\right)=-\frac{1}{3}\left(k_{11} \frac{d g^{11}}{d t}+2 k_{22} \frac{d g^{22}}{d t}+g^{11} \frac{d k_{11}}{d t}+2 g^{22} \frac{d k_{22}}{d t}\right) \\
& =-\frac{1}{3}\left(R+H^{2}+\frac{T_{11}^{1}}{a^{2}}+\frac{T_{11}^{2}}{a^{2}}+2 \frac{T_{22}^{1}}{b^{2}}+2 \frac{T_{22}^{2}}{b^{2}}+\frac{\tau_{11}}{a^{2}}+2 \frac{\tau_{22}}{b^{2}}-12 \pi\left(T_{00}^{1}+\tau_{00}+T_{00}^{2}+H_{00}\right)-3 \Lambda\right) \\
& \frac{d H}{d t}=-\frac{1}{3}\left(R+(t r k)^{2}+4 \pi g^{i j}\left(T_{i j}^{1}+T_{i j}^{2}\right)-12 \pi\left(T_{00}^{1}+T_{00}^{2}+H_{00}\right)-8 \pi \tau_{00}-3 \Lambda\right)
\end{aligned}
$$

Where $R=g^{i j} R_{i j}$. Using the Hamiltonian constraint, we have:

$$
R+(t r k)^{2}=k_{i j} k^{i j}+2 \Lambda+16 \pi\left(T_{00}^{1}+T_{00}^{2}+H_{00}+\tau_{00}\right) .
$$

Replacing (62) in (61), we then have

$$
\frac{d H}{d t}=-\frac{1}{3}\left(k_{i j} k^{i j}-\Lambda+4 \pi g^{i j}\left(T_{i j}^{1}+T_{i j}^{2}\right)+4 \pi\left(T_{00}^{1}+T_{00}^{2}+H_{00}+2 \pi \tau_{00}\right) .\right.
$$

Introduce the traceless tensor associated to $k_{i j}: L_{i j}=k_{i j}+g_{i j} H$. By a direct calculation, we get 


$$
k_{i j} k^{i j}=3 H^{2}+L_{i j} L^{i j}
$$

Using (64) in(63), we obtain

$$
\frac{d H}{d t}=-\frac{1}{3}\left(3 H^{2}+L_{i j} L^{i j}-\Lambda+4 \pi g^{i j}\left(T_{i j}^{1}+T_{i j}^{2}\right)+4 \pi\left(T_{00}^{1}+T_{00}^{2}+H_{00}\right)+8 \pi \tau_{00}\right) .
$$

That gives (60). +

Lemma 3: Let $\Lambda \geq 0$ be given, and suppose $H(0)>0$; then $H$ is uniformly bounded and we have:

$$
H \in\left[H_{0}^{1}=\sqrt{\frac{\Lambda}{3}}, H(0)\right]
$$

proof: Recall that $\frac{d H}{d t}$ is given by (60), but since

$$
L_{i j} L^{i j}>0, g^{i j} \tau_{i j}=\tau_{00}>0, T_{00}^{1}>0, H_{00}>0, T_{00}^{2}>0, \psi>0
$$

then (60) yield:

$$
\frac{d H}{d t} \leq \frac{1}{3}\left(-3 H^{2}+\Lambda+4 \pi m_{0}^{2} \phi^{2}\right)
$$

Using (60) and (61), we get

$$
-6 H^{2}+2 \Lambda+8 \pi m_{0}^{2} \phi^{2}=-L_{i j} L^{i j}-16 \pi\left(T_{00}^{1}+\tau_{00}+H_{00}\right)+R-16 \pi \psi
$$

But it is proved (see [7] ) that $R \leq 0$. Then

$$
-3 H^{2}+\Lambda \leq 0
$$

Now, we then obtain

$$
H^{2} \geq \frac{\Lambda}{3}
$$

which implies

$$
H \geq \sqrt{\frac{\Lambda}{3}} \text { or } H \leq-\sqrt{\frac{\Lambda}{3}} .
$$

By hypothesis, (66) and (67), $\quad H>0$ and $\frac{d H}{d t} \leq 0$ then

$$
\sqrt{\frac{\Lambda}{3}} \leq H \leq H(0)+
$$

Remark 3: Domain of the variables $H, s, z, \Sigma_{+}$.

One easily observes that the variables $H, s, z, \Sigma_{+}$will be taken on the subset $\mathrm{D}$ of $\mathrm{R}^{4}$ defined by:

$$
D=\begin{gathered}
\left\{\left(H, s, z, \Sigma_{+}\right) \in \mathrm{R}^{4} \text { suchthat } H_{1}^{0}<H \leq H_{o}\right. \\
\left.0<s<1 ; 0<z<1 ;-1<\Sigma_{+}<1\right\}
\end{gathered}
$$


In what follows, we assume $\Lambda \geq 0$ and $\dot{b}_{0}>0, f \in H_{d, r}^{3}\left(O, T, \mathrm{R}^{3}\right)$ is also fixed. We are looking for a solution $\left(H, s, z, \Sigma_{+}\right)$of (56)-(59), on the interval $I=\left[t_{0}, t_{0}+T\right], T>0$, which satisfies at the initial instant $t=t_{0}$ the condition $\left(H, s, z, \Sigma_{+}\right)\left(t_{0}\right)=\left(H_{t_{0}}, s_{t_{0}}, z_{t_{0}}, \Sigma_{+t_{0}}\right)$, where $H_{t_{0}}, s_{t_{0}}, z_{t_{0}}, \Sigma_{+t_{0}}$ are real numbers conveniently fixed, in fact we must return to definitions of $H, s, z, \Sigma_{+}$, and choose those data according to $a_{t_{0}}, b_{t_{0}}, \dot{a}_{t_{0}}, \dot{b}_{t_{0}}$ (and $\left.\phi_{t_{0}}, \psi_{t_{0}}, E^{i}, \varphi_{i j}\right)$ and, furthermore subject to Hamiltonian constraint (40).

Definition 2: If $x$ is a real number such that $x \in] 0,1[$, we set

$$
\beta(x)=\inf (x, 1-x) \text {. }
$$

Remark 4: Since $\mathrm{H}_{0}>0$, we assume that $\dot{a}_{0}>0$. So the initial data $a_{0}, b_{0}, \dot{a}_{0}, \dot{b}_{0}$ will be taken such that $a_{0}>0, b_{0}>0, \dot{b}_{0}>0$ and $\dot{a}_{0}>0$.

We now prove the local existence theorem of solutions to system (56), (57), (58) and (59) with the initial datum $\left(H, s, z, \Sigma_{+}\right)\left(t_{0}\right)=\left(H_{t_{0}}, s_{t_{0}}, z_{t_{0}}, \Sigma_{+t_{0}}\right) \in D$.

Proposition 5: Let $\delta>0, t_{0} \geq 0$ be given, then any solution $\left(H, s, z, \Sigma_{+}\right)$of system (56), (57), (58) and (59) on $\left[t_{0}, t_{0}+T\right]$ verifies the following inequalities for all $t \in[0, \delta]$

$$
\begin{gathered}
\frac{1}{H\left(t_{0}+t\right)} \leq \frac{1}{H\left(t_{0}\right)} e^{3 H_{0}(T+\delta)} \leq \gamma_{0} e^{6 H_{0}(T+\delta)} \\
\frac{1}{\beta\left(s\left(t_{0}+t\right)\right)} \leq \frac{1}{\beta\left(s\left(t_{0}\right)\right)} e^{6 H_{0}(T+\delta)} \leq \gamma_{0} e^{6 H_{0}(T+\delta)} \\
\frac{1}{\beta\left(z\left(t_{0}+t\right)\right)} \leq \frac{1}{\beta\left(z\left(t_{0}+t\right)\right)} e^{10 H_{0}(T+\delta)} \leq \gamma_{0} e^{10 H_{0}(T+\delta)}
\end{gathered}
$$

where

$$
\gamma_{0}=\left(\frac{1}{H_{0}}+\frac{1}{\beta\left(s_{0}\right)}+\frac{1}{\beta\left(z_{0}\right)}\right)
$$

Proof see $[1]+$

In what follows, $C>0$ is a constant. We will apply the standard theory on the first order differential systems. With a view to succeed. We will study the function $Z$ defined using the r.h.s of system (56), (57), (58) and (59) by

$$
Z\left(t, H, s, z, \Sigma_{+}\right)=\left(Z_{1}, Z_{2}, Z_{3}, Z_{4}\right)\left(t, H, s, z, \Sigma_{+}\right) \text {. }
$$

We recall that $Z$ is defined on $\mathrm{D}$ defined by (68). We must prove that $Z$ is a continuous function of $t$, locally Lipschitzian in $X=\left(H, s, z, \Sigma_{+}\right) \in \mathrm{R}^{4}$ with the norm

$$
\|X\|_{1}=|H|+|s|+|z|+\left|\Sigma_{+}\right|
$$

$Z$ is obviously a continuous function of t on one hand, on the other hand, $Z_{2}$ and $Z_{3}$ are polynomial function in $H, s, z$ and $\Sigma_{+}$, so locally Lipschitzian. 
Concerning now $Z_{1}$ and $Z_{4}$, we need some energy estimations

lemma 4: Let $\left.s_{1}, s_{2}, z_{1}, z_{2} \in\right] 0,1\left[, f \in H_{d, r}^{3}\left(t_{0}, t_{0}+\delta, \mathrm{R}^{3}\right), H_{1}, H_{2} \in\right] H_{1}^{0}, H_{0}[$. Then one has for $i=1,2$ :

$$
\begin{aligned}
& \left\{\begin{array}{c}
\left|\frac{1}{p^{0}\left(s_{1}, z_{1}, \bar{p}\right)}-\frac{1}{p^{0}\left(s_{2}, z_{2}, \bar{p}\right)}\right| \leq \frac{C\left[\left|s_{1}-s_{2}\right|+\left|z_{1}-z_{2}\right|\right]}{\beta^{\frac{3}{2}}\left(s_{2}\right) \beta\left(z_{1}\right) \beta\left(z_{2}\right)} \times \frac{1}{\left|p^{i}\right|}, \\
\left|\frac{1}{p^{0}\left(s_{1}, z_{1}, \bar{p}\right)}-\frac{1}{p^{0}\left(s_{2}, z_{2}, \bar{p}\right)}\right| \leq \frac{C\left[s_{1}-s_{2}|+| z_{1}-z_{2} \mid\right]}{\beta^{\frac{3}{2}}\left(s_{2}\right) \beta\left(z_{1}\right) \beta\left(z_{2}\right)} \times \frac{1}{\left|p^{0}\left(s_{1}, z_{1}, \bar{p}\right)\right|}, \\
\frac{1}{p^{0}\left(s_{1}, z_{1}, \bar{p}\right)}-\frac{1}{P^{p 0}\left(s_{2}, z_{2}, \bar{p}\right)} \mid \leq \frac{C\left[\left|s_{1}-s_{2}\right|+\left|z_{1}-z_{2}\right|\right]}{\beta^{\frac{3}{2}}\left(s_{2}\right) \beta\left(z_{1}\right) \beta\left(z_{2}\right)} \times \frac{1}{\mid p^{0}\left(s_{2}, z_{2}, \bar{p}\right)},
\end{array}\right. \\
& \left\{\begin{array}{c}
\left|P_{i}\left(s_{1}, z_{1}, f, \phi, \psi\right)-P_{i}\left(s_{2}, z_{2}, f, \phi, \psi\right)\right| \leq \frac{C\left[1+\|f(t)\| \mid\left(\left|s_{1}-s_{2}\right|+\left|z_{1}-z_{2}\right|\right)\right.}{\beta^{4}\left(s_{1}\right) \beta^{4}\left(s_{2}\right) \beta^{6}\left(z_{1}\right) \beta^{4}\left(z_{2}\right)}, \\
\left|P_{i}(s, z, f, \phi, \psi)\right| \leq \frac{C(1+\|f(t)\|)}{\beta^{\frac{5}{2}}(s) \beta^{3}(z)}, \\
\left|\frac{P_{i}\left(s_{1}, z_{1}, f, \phi, \psi\right)}{H_{1}}-\frac{P_{i}\left(s_{2}, z_{2}, f, \phi, \psi\right)}{H_{2}}\right| \leq \frac{C\left[1+\|f(t)\|||\left(\left|H_{1}-H_{2}\right|+\left|s_{1}-s_{2}\right|+\left|z_{1}-z_{2}\right|\right)\right.}{H_{1} H_{2} \beta^{4}\left(s_{1}\right) \beta^{4}\left(s_{2}\right) \beta^{6}\left(z_{1}\right) \beta^{4}\left(z_{2}\right)},
\end{array}\right.
\end{aligned}
$$

Where $p^{0}, P_{i}$ are given by (51) and (52).

Proof: see [6].+

Using lemma 4, we can write

$$
\begin{aligned}
& \left\|Z\left(H_{1}, s_{1}, z_{1}, \Sigma_{+1}\right)-Z\left(H_{2}, s_{2}, z_{2}, \Sigma_{+2}\right)\right\|_{1} \leq N\left\|\left(H_{1}, s_{1}, z_{1}, \Sigma_{+1}\right)-\left(H_{2}, s_{2}, z_{2}, \Sigma_{+2}\right)\right\|_{1} . \\
& \text { where } \\
& N=\frac{C(1+\|f(t)\|)}{H_{1} H_{2} \beta^{4}\left(s_{1}\right) \beta^{4}\left(s_{2}\right) \beta^{6}\left(z_{1}\right) \beta^{4}\left(z_{2}\right)}
\end{aligned}
$$

Proposition 6: Let $f \in H_{d, r}^{3}\left(t_{0}, t_{0}+T, \mathrm{R}^{3}\right), T>0$, then system (56), (57), (58) and (59) with the initial datum $\left(H_{t_{0}}, s_{t_{0}}, z_{t_{0}}, \Sigma_{+t_{0}}\right)$ at $t=t_{0}$ verifying (68) has a unique solution $\left(H, s, z, \Sigma_{+}\right)$on $\left[t_{0}, t_{0}+T\right]$ such that $\left(H, s, z, \Sigma_{+}\right)\left(t_{0}\right)=\left(H_{t_{0}}, s_{t_{0}}, z_{t_{0}}, \Sigma_{+t_{0}}\right)$.

Proof: Let $f \in H_{d, r}^{3}\left(t_{0}, t_{0}+T, \mathrm{R}^{3}\right), T>0$ and $\left(H^{0}, s^{0}, z^{0}, \Sigma_{+}^{0}\right) \in D$, be given. Now consider the neighborhood $\mathrm{W}$ of $\left(H^{0}, s^{0}, z^{0}, \Sigma_{+}^{0}\right)$ defined by $\left.W=\right] H_{1}^{0}, H^{0}[\times] \frac{s^{0}}{2}, \frac{s^{0}+1}{2}[\times] \frac{z^{0}}{2}, \frac{z^{0}+1}{2}[\times]-1,1[$ If we take $\left(H_{1}, s_{1}, z_{1}, \Sigma_{+1}\right)$ and $\left(H_{2}, s_{2}, z_{2}, \Sigma_{+2}\right) \in W$, then

$$
\frac{1}{H_{i}} \leq \frac{1}{H_{1}^{0}}, \frac{1}{\beta\left(s_{i}\right)} \leq \frac{2}{\beta\left(s^{0}\right)}, \frac{1}{\beta\left(z_{i}\right)} \leq \frac{2}{\beta\left(z^{0}\right)}, i=1,2
$$

Using (76)-(78), we get 


$$
\left\|Z\left(H_{1}, s_{1}, z_{1}, \Sigma_{+1}\right)-Z\left(H_{2}, s_{2}, z_{2}, \Sigma_{+2}\right)\right\|_{1} \leq N_{0}\left\|\left(H_{1}, s_{1}, z_{1}, \Sigma_{+1}\right)-\left(H_{2}, s_{2}, z_{2}, \Sigma_{+2}\right)\right\|
$$

where

$$
N_{0}=\frac{C r}{\left(H_{1}^{0}\right)^{2} \beta^{8}\left(s^{0}\right) \beta^{10}\left(z^{0}\right)},
$$

Consequently, the function $Z$ defined by (73) is locally Lipschitzian and uniformly bounded, so by the standard theory on the first order differential system, we conclude that system "(56)-(59)" has a unique solution $\left(H, s, z, \Sigma_{+}\right)$on $\left[t_{0}, t_{0}+T\right]$ which verifies at $t=t_{0}:\left(H, s, z, \Sigma_{+}\right)\left(t_{0}\right)=\left(H_{t_{0}}, s_{t_{0}}, z_{t_{0}}, \Sigma_{+t_{0}}\right) .+$

Theorem 2: Let $r>0, d>\frac{5}{2}, \quad f \in H_{d, r}^{3}\left(t_{0}, t_{0}+T, \mathrm{R}^{3}\right), E^{i} \in \mathrm{R}, \phi_{0}, \psi_{0} \in \mathrm{R}, \varphi_{i j} \in \mathrm{R}, T>0$ be given, Let $a_{t_{0}}>0, b_{t_{0}}>0, \dot{b}_{t_{0}}, \quad \dot{a}_{t_{0}}, \Lambda \geq 0$ verify the constraint of remark 4 such that the whole system satisfying the constraints system " (40)-(44)". Then the Cauchy problem for Einstein system (33)-(35) with the cosmological constant has a unique solution $(a, b)$ on $\left[t_{0}, t_{0}+T\right]$, such that $\left\{\begin{array}{l}(a, b)\left(t_{0}\right)=\left(a_{t_{0}}, b_{t_{0}}\right) \\ (\dot{a}, \dot{b})\left(t_{0}\right)=\left(\dot{a}_{t_{0}}, \dot{b}_{t_{0}}\right)\end{array}\right.$.

Proof: Using the change of variables (47), the system (33)-(35) is equivalent to the system "(56)-(59)", applying proposition 6 , the system "(56)-(59)", has a unique solution $\left(H, s, z, \Sigma_{+}\right)$on $\left[t_{0}, t_{0}+T\right]$ if the initial datum $\left(H_{t_{0}}, s_{t_{0}}, z_{t_{0}}, \Sigma_{+t_{0}}\right)$ verifies (68). Taking at $t=t_{0}$, the initial data $H_{t_{0}}, s_{t_{0}}, z_{t_{0}}, \Sigma_{+_{0}}$, such that (47) hold, we realize that $\left(H_{t_{0}}, s_{t_{0}}, z_{t_{0}}, \Sigma_{+t_{0}}\right) \in D$, Consequently, system (56)-(59), has of course, a unique solution $\left(H, s, z, \Sigma_{+}\right)$on $\left[t_{0}, t_{0}+T\right]$ with the initial data $H_{t_{0}}, s_{t_{0}}, z_{t_{0}}, \Sigma_{+t_{0}}$ at $\mathrm{t}=t_{0}$. Relations (49) solved in $a^{2}$ and $b^{2}$ then give the unique solution $(a, b)$ of system (33)-(35).

Corollary 1: For the solution $(a, b)$ to system (33)-(35) on $\left[t_{0}, t_{0}+T\right], t_{0} \in \mathrm{R}, T \geq 0$, the map $t \mapsto b(t)$ is increasing and there exists $\mathrm{C}_{0}>0$ such that $\left|\frac{\dot{a}}{a}\right| \leq C_{0}$ and $\left|\frac{\dot{b}}{b}\right| \leq C_{0}$.

Proof: we have following (55): $\frac{\dot{a}}{a}=H\left(1-2 \Sigma_{+}\right), \frac{\dot{b}}{b}=H\left(1+\Sigma_{+}\right) \geq 0, b>0$, then

$$
\left|\frac{\dot{a}}{a}\right|=\left|H \|\left(1-2 \Sigma_{+}\right)\right| \leq 2 H_{0} \cdot\left|\frac{\dot{b}}{b}\right|=|H|\left(1+\Sigma_{+}\right) \leq 2 H_{0} .+
$$

Remark5: we then deduce from corollary 1 that: $a(t) \leq a_{0} e^{K_{0} t}, b(t) \leq b_{0} e^{K_{0} t}, \frac{1}{a}(t) \leq \frac{1}{a_{0}} e^{K_{0} t}, \frac{1}{b}(t) \leq \frac{1}{b_{0}} e^{K_{0} t}$.

\section{LOCAL EXISTENCE OF SOLUTION FOR THE COUPLED SYSTEM}

we are searching in the case $\Lambda \geq 0$, the local solution to the system.

The coupled system, reduces to the following system, in which $a, b, a^{2}, b^{2}, \frac{\dot{a}}{a}, \frac{\dot{b}}{b}$, defined by formulas (47) and (48) are solved in $H, s, z, \Sigma_{+}$: 


$$
\begin{aligned}
& \frac{d H}{d t}=-\frac{3}{2}\left(1+\Sigma_{+}^{2}\right) H^{2}-\frac{P_{1}+2 P_{2}}{6}+\frac{\Lambda}{2} \\
& \frac{d s}{d t}=6 s(1-s) \Sigma_{+} H \\
& \frac{d z}{d t}=2 z(1-z)\left(1+\Sigma_{+}-3 s \Sigma_{+}\right) H \\
& \frac{d \Sigma_{+}}{d t}=-\frac{3}{2}\left(1-\Sigma_{+}^{2}\right) H \Sigma_{+}+\frac{P_{1}}{6 H}\left(\Sigma_{+}-2\right)+\frac{P_{2}}{3 H}\left(\Sigma_{+}+1\right)-\frac{\Lambda \Sigma_{+}}{2 H} . \\
& \frac{d \phi}{d t}=\sqrt{2 \psi} \\
& \frac{d \psi}{d t}=-6 H \psi-m_{0}^{2} \phi \sqrt{2 \psi}-1 \\
& \frac{d f}{d t}=\frac{1}{p^{o}} Q(f, f, \bar{p}) \\
& \text { (S) } \\
& \frac{d p^{1}}{d t}=-2 H\left(1-2 \Sigma_{+}^{2}\right) p^{1}-a_{0} b_{0}^{2} E^{1} \int_{\mathrm{R}^{3}} f d \bar{p} \\
& -\frac{2 \sqrt{s z}\left(p^{2} \varphi_{12}+p^{3} \varphi_{13}\right) \int_{\mathrm{R}^{3}} f d \bar{p}}{\left.(1-s) \sqrt{1-z} \sqrt{s(1-s)(1-z)+z\left[(1-s)\left(p^{1}\right)^{2}+2 s\left(\left(p^{2}\right)^{2}+\left(p^{3}\right)^{2}\right)\right.}\right]}, \\
& \frac{d p^{2}}{d t}=-2 H\left(1+\Sigma_{+}^{2}\right) p^{2}-a_{0} b_{0}^{2} E^{2} \int_{\mathrm{R}^{3}} f d \bar{p} \\
& +\frac{2 \sqrt{z}\left(p^{1} \varphi_{21}+p^{3} \varphi_{23}\right) \int_{\mathrm{R}^{3}} f d \bar{p}}{\sqrt{s(1-z)} \sqrt{s(1-s)(1-z)+z\left[(1-s)\left(p^{1}\right)^{2}+2 s\left(\left(p^{2}\right)^{2}+\left(p^{3}\right)^{2}\right)\right]}}, \\
& \frac{d p 3}{d t}=-2 H\left(1+\Sigma_{+}^{2}\right) p^{3}-a_{0} b_{0}^{2} E^{3} \int_{\mathrm{R}^{3}} f d \bar{p} \\
& -\frac{2 \sqrt{z}\left(p^{1} \varphi_{32}+p^{2} \varphi_{32}\right) \int_{\mathrm{R}^{3}} f d \bar{p}}{\sqrt{s(1-z)} \sqrt{s(1-s)(1-z)+z\left[(1-s)\left(p^{1}\right)^{2}+2 s\left(\left(p^{2}\right)^{2}+\left(p^{3}\right)^{2}\right)\right]}} \\
& F^{0 i}=\frac{a_{0} b_{0}^{2} E^{i}}{a b^{2}} \quad F_{i j}=\varphi_{i j}, \quad i, j=1,2,3
\end{aligned}
$$

Let us set $\mathrm{X}=\left(H, s, z, \Sigma_{+}, \phi, \psi, f, \bar{p}\right), X(0)=X_{0}$ and $X\left(t_{0}\right)=X_{t_{0}}$. 


$$
E=\mathrm{R}^{4} \times H_{d}^{3}\left(\mathrm{R}^{3}\right) \times \mathrm{R}^{2} \times \mathrm{R}^{3}, d>\frac{5}{2} \text { endowed with the norm }
$$

$$
\|X\|_{E}=|H|+|s|+|z|+\left|\Sigma_{+}\right|+\|f\|+|\phi|+|\psi|+\|\bar{p}\|_{\mathrm{R}^{3}}
$$

We will show that the map $h(X)=\left(\square_{Z_{1}}, \square_{2}, \square_{3}, \square_{4}, h_{1}, h_{2}, h_{3}, h_{4}, h_{5}, h_{6}\right)$ defined by the r.h.s of system (S) defined by (80) is continuous of $t$ and localy Lipschitzian in $X \in \mathrm{E}$ endowed with the norm (81).

We also need this time to compute the differences in $f$ and those in s and $z$ in $\frac{1}{p^{o}} Q(f, f, \bar{p})$. We must prove that there exists some $\delta>0$ such that system (S) has a unique solution $X$ defined on $I_{0}=[0, \delta]$ and taking at $t=0$, the initial datum $X_{0}$ deduce from the initial data $a_{0}, b_{0}, \dot{a}_{0}, \dot{b}_{0}$, using formulas (47) . Let us recall that the initial data $a_{0}, b_{0}, \dot{a}_{0}, \dot{b}_{0}, \phi_{0}, \psi_{0}, f_{0}, \bar{p}_{0}, E^{i}, \varphi_{i j}$ are subject to the constraints (40)-(41)-(42)-(44).

The following energy estimations shall be useful in what is to follow.

Lemma 5: Let $\left.s_{1}, s_{2}, z_{1}, z_{2} \in\right] 0 ; 1[, a, b$ defined by (49) and $g$ defined by (1) be given. Then one has:

$$
\begin{gathered}
\left|a b^{2}\left(s_{1}, z_{1}\right)-a b^{2}\left(s_{2}, z_{2}\right)\right| \leq \frac{C\left[\left|s_{1}-s_{2}\right|+\left|z_{1}-z_{2}\right|\right]}{\beta^{2}\left(s_{1}\right) \beta^{\frac{3}{2}}\left(s_{2}\right) \beta^{3}\left(z_{1}\right) \beta^{\frac{3}{2}}\left(z_{2}\right)} \\
\left|a b^{2} g^{11}\left(s_{1}, z_{1}\right)-a b^{2} g^{11}\left(s_{2}, z_{2}\right)\right| \leq \frac{C\left[\left|s_{1}-s_{2}\right|+\left|z_{1}-z_{2}\right|\right]}{\beta\left(s_{1}\right) \beta\left(s_{2}\right) \beta\left(z_{1}\right) \beta^{\frac{1}{2}}\left(z_{2}\right)} \\
\left|a b^{2} g^{22}\left(s_{1}, z_{1}\right)-a b^{2} g^{22}\left(s_{2}, z_{2}\right)\right| \leq \frac{C\left[\left|s_{1}-s_{2}\right|+\left|z_{1}-z_{2}\right|\right]}{\beta\left(s_{1}\right) \beta\left(s_{2}\right) \beta\left(z_{1}\right) \beta^{\frac{1}{2}}\left(z_{2}\right)} \\
\left|a^{2}\left(s_{1}, z_{1}\right)-a^{2}\left(s_{2}, z_{2}\right)\right| \leq \frac{C\left[\left|s_{1}-s_{2}\right|+\left|z_{1}-z_{2}\right|\right]}{\beta\left(s_{1}\right) \beta\left(s_{2}\right) \beta\left(z_{1}\right) \beta\left(z_{2}\right)} \\
\left|b^{2}\left(s_{1}, z_{1}\right)-b^{2}\left(s_{2}, z_{2}\right)\right| \leq \frac{C\left[\left|s_{1}-s_{2}\right|+\left|z_{1}-z_{2}\right|\right]}{\beta\left(s_{1}\right) \beta\left(s_{2}\right) \beta\left(z_{1}\right) \beta\left(z_{2}\right)}
\end{gathered}
$$




$$
\left|B\left(s_{1}, z_{1}, \bar{p}, \bar{q}, \bar{p}, \bar{q}^{\prime}\right)-B\left(s_{2}, z_{2}, \bar{p}, \bar{q}, \bar{p}, \bar{q}^{\prime}\right)\right| \leq \frac{3 K_{0}}{\beta^{\frac{3}{2}}\left(z_{1}\right) \beta\left(s_{2}\right) \beta^{\frac{1}{2}}\left(s_{1}\right) \beta\left(z_{2}\right)} C\left[\left|s_{1}-s_{2}\right|+\left|z_{1}-z_{2}\right|\right] \text { (82) }
$$

Proof: Apart from (82), the five first inequalities are just direct computation. Now for (82), recall that we require the boundedness and Lipschitz continuity assumption on B. We then obtain (82) by direct computation. +

Proposition 7: Let $\left.\bar{p}_{j}=\left(p_{j}^{i}\right) \in \mathrm{R}^{3}, f, f_{j} \in H_{d}^{3}\left(\mathrm{R}^{3}\right), s_{j}, z_{j} \in\right] 0 ; 1[$ for $\mathrm{j}=1,2$ be given. Then

$$
\begin{aligned}
& \left\|\left(\frac{1}{p^{0}\left(s_{1}, z_{1}\right)}-\frac{1}{p^{0}\left(s_{2}, z_{2}\right)}\right) Q\left(f_{1}, f_{1}\right)\left(s_{2}, z_{2}\right)\right\| \leq \frac{C\left\|f_{1}\right\|^{2}\left[\left|s_{1}-s_{2}\right|+\left|z_{1}-z_{2}\right|\right]}{\beta^{\frac{7}{2}}\left(s_{2}\right) \beta\left(z_{1}\right) \beta^{\frac{5}{2}}\left(z_{2}\right)} . \\
& \left\|\frac{1}{p^{0}\left(s_{2}, z_{2}\right)}\left(Q\left(f_{1}, f_{1}\right)\left(s_{1}, z_{1}\right)-Q\left(f_{2}, f_{2}\right)\left(s_{2}, z_{2}\right)\right)\right\| \leq \frac{C\left[\left\|f_{1}\right\|+\left\|f_{2}\right\|\right]|| f_{1}-f_{2} \|}{\beta^{\frac{3}{2}}\left(s_{2}\right) \beta^{\frac{3}{2}}\left(z_{2}\right)} . \\
& \left\|\frac{1}{p^{0}\left(s_{1}, z_{1}\right)}\left(Q(f, f)\left(s_{1}, z_{1}\right)-Q(f, f)\left(s_{2}, z_{2}\right)\right)\right\| \leq \frac{C|| f_{1} \|^{2}\left[\left|s_{1}-s_{2}\right|+\left|z_{1}-z_{2}\right|\right]}{\beta^{2}\left(s_{1}\right) \beta^{\frac{7}{2}}\left(s_{2}\right) \beta^{3}\left(z_{1}\right) \beta^{\frac{5}{2}}\left(z_{2}\right)} . \\
& \left\|\frac{a b^{2} g^{i i}\left(s_{1}, z_{1}\right) \varphi_{i k} p_{1}^{k}}{p^{0}\left(s_{1}, z_{1}, \bar{p}_{1}\right)} \int f_{1} d \bar{p}-\frac{a b^{2} g^{i i}\left(s_{2}, z_{2}\right) \varphi_{i k} p_{2}^{k}}{p^{0}\left(s_{2}, z_{2}, \bar{p}_{2}\right)} \int f_{2} d \bar{p}\right\| \leq \\
& \frac{C\left[1+\left\|f_{1}\right\|\right\}\left(\left\|f_{1}-f_{2}\right\|+\left|s_{1}-s_{2}\right|+\left|z_{1}-z_{2}\right|+|| \bar{p}_{1}-\bar{p}_{2} \|\right)}{\beta^{2}\left(s_{1}\right) \beta^{\frac{3}{2}}\left(s_{2}\right) \beta^{4}\left(z_{1}\right) \beta^{\frac{3}{2}}\left(z_{2}\right)} . \\
& \|\left(\frac{1}{p^{0}\left(s_{1}, z_{1}, \bar{p}_{1}\right)} Q\left(f_{1}, f_{1}\right)\left(s_{1}, z_{1}, \bar{p}_{1}\right)-\frac{1}{p^{0}\left(s_{2}, z_{2}, \bar{p}_{2}\right)} Q\left(f_{2}, f_{2}\right)\left(s_{2}, z_{2}, \bar{p}_{2}\right) \| \leq\right. \\
& \frac{C\left[1+\left\|f_{1}\right\|+\left\|f_{2}\right\|+\left\|f_{1}\right\|^{2}+\left\|f_{2}\right\|^{2}\left(\left\|f_{1}-f_{2}\right\|+\left|s_{1}-s_{2}\right|+\left|z_{1}-z_{2}\right|+|| \bar{p}_{1}-\bar{p}_{2} \|\right)\right.}{\beta^{2}\left(s_{1}\right) \beta^{\frac{7}{2}}\left(s_{2}\right) \beta^{3}\left(z_{1}\right) \beta^{\frac{5}{2}}\left(z_{2}\right)} .
\end{aligned}
$$

Proof: see [6] Concerning the differences in $P_{i}$ and $\frac{P_{i}}{H}, i=1,2$, we have the following result which is a direct consequence of the Lemma 5. 
Proposition 8: Let $\left.s_{1}, s_{2}, z_{1}, z_{2} \in\right] 0 ; 1\left[, f_{1}, f_{2} \in H_{d}^{3}\left(\mathrm{R}^{3}\right), H_{1}, H_{2} \in\right] H_{1}^{0}, H_{0}\left[, \phi_{1}, \phi_{2}, \psi_{1}, \psi_{2} \in \mathrm{R}\right.$ be given; then for $i=1,2$

we have:

$$
\begin{gathered}
\left|P_{i}\left(s_{1}, z_{1}, f_{1}, \phi_{1}, \psi_{1}\right)-P_{i}\left(s_{2}, z_{2}, f_{2}, \phi_{2}, \psi_{2}\right)\right| \leq \\
M_{2}\left[|| f_{1}-f_{2}||+\left|\phi_{1}-\phi_{2}\right|+\left|\psi_{1}-\psi_{2}\right|+\left|s_{1}-s_{2}\right|+\left|z_{1}-z_{2}\right|\right] \\
\left|\frac{P_{i}\left(s_{1}, z_{1}, f_{1}, \phi_{1}, \psi_{1}\right)}{H_{1}}-\frac{P_{i}\left(s_{2}, z_{2}, f_{2}, \phi_{2}, \psi_{2}\right)}{H_{2}}\right| \leq \\
M_{3}\left[\left|H_{1}-H_{2}\right|+\left|s_{1}-s_{2}\right|+\left|z_{1}-z_{2}\right|+|| f_{1}-f_{2}|+| \phi_{1}-\phi_{2}|+| \psi_{1}-\psi_{2} \mid\right]
\end{gathered}
$$

Where

$$
\begin{gathered}
M_{2}=\frac{C\left(1+\left\|f_{1}\right\|+\left\|f_{2}\right\|\right)}{\beta^{4}\left(s_{1}\right) \beta^{4}\left(s_{2}\right) \beta^{6}\left(z_{1}\right) \beta^{4}\left(z_{2}\right)}+1+m_{0}^{2}\left|\phi_{1}+\phi_{2}\right| \\
M_{3}=\frac{C\left(3+\left\|f_{1}\right\|+\left\|f_{2}\right\|\right)}{H_{1} H_{2} \beta^{4}\left(s_{1}\right) \beta^{4}\left(s_{2}\right) \beta^{6}\left(z_{1}\right) \beta^{4}\left(z_{2}\right)}+1+m_{0}^{2}\left|\phi_{1}+\phi_{2}\right|
\end{gathered}
$$

proof: Considering the fact that $\left.f, f_{i} \in H_{d}^{3}\left(\mathrm{R}^{3}\right), s_{i}, z_{i} \in\right] 0 ; 1\left[, H_{i} \in\right] H_{1}^{0}, H_{0}\left[, \phi_{i}, \psi_{i} \in \mathrm{R}\right.$, for $i=1,2$ yield to:

$$
\begin{aligned}
& P_{i}\left(s_{1}, z_{1}, f_{1}, \phi_{1}, \psi_{1}\right)-P_{i}\left(s_{2}, z_{2}, f_{2}, \phi_{2}, \psi_{2}\right)= \\
& 16 \pi i\left(\theta_{1}-\theta_{2}\right) \int_{\mathrm{R}^{3}} \frac{\left(p^{1}\right)^{2} f_{1}}{p^{0}\left(z_{1}, s_{1}\right)} d \bar{p}+16 \pi i \theta_{2} \int_{\mathrm{R}^{3}} \frac{1}{p^{0}\left(z_{1}, s_{1}\right)}\left(p^{i}\right)^{2}\left(f_{1}-f_{2}\right) d \bar{p}+ \\
& 16 \pi i \theta_{2} \int_{\mathrm{R}^{3}}\left(\frac{1}{p^{0}\left(s_{1}, z_{1}\right)}-\frac{1}{p^{0}\left(s_{2}, z_{2}\right)}\right) f_{2} d \bar{p}+K_{1}\left(V_{1}-V_{2}\right)+ \\
& K_{i}\left(W_{1}-W_{2}\right)+\left|\psi_{1}-\psi_{2}\right|+m_{0}^{2}\left|\phi_{1}-\phi_{2}\right|\left|\phi_{1}+\phi_{2}\right|
\end{aligned}
$$

where

$$
\left\{\begin{array}{c}
\theta_{i}=\frac{1}{s_{i}^{\left(\frac{5}{2}-i\right)}\left(1-s_{i}\right)^{i}}\left(\frac{z_{i}}{1-z_{i}}\right)^{\frac{5}{2}}, X_{i}=\frac{1}{s_{i}^{\frac{1}{2}}\left(1-s_{i}\right)^{2}}\left(\frac{z_{i}}{1-z_{i}}\right)^{\frac{5}{2}} \\
V_{i}=\frac{\left(1-s_{i}\right)^{2}\left(1-z_{i}\right)^{2}}{z_{i}^{2}}, W_{i}=\frac{s_{i}\left(1-s_{i}\right)\left(1-z_{i}\right)^{2}}{z_{i}^{2}}, K_{1}=-\pi\left(\left(a_{0} b_{0}^{2} E^{1}\right)^{2}+\varphi_{23}^{2}\right), \\
K_{i}=2 \pi\left((-1)^{i+1}\left(a_{0} b_{0}^{2} E^{2}\right)^{2}+\left(a_{0} b_{0}^{2} E^{3}\right)^{2}+\varphi_{12}^{2}+(-1)^{i+1} \varphi_{13}^{2}\right),
\end{array}\right.
$$


Inequalities (74) yield to:

$$
\left|\frac{1}{p^{0}\left(s_{1}, z_{1}, \bar{p}\right)}-\frac{1}{p^{0}\left(s_{2}, z_{2}, \bar{p}\right)}\right| \leq \frac{C\left[s_{1}-s_{2}|+| z_{1}-z_{2} \mid\right]}{\beta^{\frac{3}{2}}\left(s_{2}\right) \beta\left(z_{1}\right) \beta\left(z_{2}\right)} \times \frac{1}{\left|p^{i}\right|}
$$

Using the lemma 4, we can see that

$$
\begin{aligned}
& \left|P_{i}\left(s_{1}, z_{1}, f_{1}, \phi_{1}, \psi_{1}\right)-P_{i}\left(s_{2}, z_{2}, f_{2}, \phi_{2}, \psi_{2}\right)\right| \leq \\
& \frac{C \| f_{1}||\left[s_{1}-s_{2}|+| z_{1}-z_{2} \mid\right]}{\beta^{4}\left(s_{1}\right) \beta^{\frac{5}{2}}\left(s_{2}\right) \beta^{\frac{11}{2}}\left(z_{1}\right) \beta^{\frac{5}{2}}\left(z_{2}\right)}+\frac{C\left\|f_{1}-f_{2}\right\|\left[\left|s_{1}-s_{2}\right|+\left|z_{1}-z_{2}\right|\right]}{\beta^{4}\left(s_{2}\right) \beta\left(z_{1}\right) \beta^{\frac{7}{2}}\left(z_{2}\right)}+ \\
& \frac{\left.C\left\|f_{2}\right\||| s_{1}-s_{2}|+| z_{1}-z_{2} \mid\right]}{\beta^{4}\left(s_{2}\right) \beta\left(z_{1}\right) \beta^{\frac{7}{2}}\left(z_{2}\right)}+\frac{\left.C\left|s_{1}-s_{2}\right|+\left|z_{1}-z_{2}\right|\right]}{\beta^{2}\left(z_{1}\right) \beta^{2}\left(z_{2}\right)}+ \\
& \left|\psi_{1}-\psi_{2}\right|+m_{0}^{2}\left|\phi_{1}-\phi_{2}\right|\left|\phi_{1}+\phi_{2}\right|,
\end{aligned}
$$

one has

$$
\begin{aligned}
& \left|\frac{P_{i}\left(s_{1}, z_{1}, f_{1}, \phi_{1}, \psi_{1}\right)}{H_{1}}-\frac{P_{i}\left(s_{2}, z_{2}, f_{2}, \phi_{2}, \psi_{2}\right)}{H_{2}}\right|= \\
& \left|\frac{1}{H_{1} H_{2}}\left(H_{2} P_{i}\left(s_{1}, z_{1}, f_{1}, \phi_{1}, \psi_{1}\right)-H_{1} P_{i}\left(s_{2}, z_{2}, f_{2}, \phi_{2}, \psi_{2}\right)\right)\right| \\
& =\mid \frac{1}{H_{1} H_{2}}\left[\left(H_{2}-H_{1}\right) P_{i}\left(s_{1}, z_{1}, f_{1}, \phi_{1}, \psi_{1}\right)+H_{1}\left(P_{i}\left(s_{1}, z_{1}, f_{1}, \phi_{1}, \psi_{1}\right)-P_{i}\left(s_{2}, z_{2}, f_{2}, \phi_{2}, \psi_{2}\right)\right) \mid\right. \\
& \leq\left[\frac{1}{H_{1} H_{2}} H_{1} \frac{C\left(1+\| f_{1} \mid\right)}{\beta^{4}\left(s_{1}\right) \beta^{4}\left(s_{2}\right) \beta^{6}\left(z_{1}\right) \beta^{4}\left(z_{2}\right)}+1+m_{0}^{2}\left|\phi_{1}+\phi_{2}\right|\right] \\
& {\left[\left|s_{1}-s_{2}\right|+\left|z_{1}-z_{2}\right|+|| f_{1}-f_{2}||+\left|\phi_{1}-\phi_{2}\right|+\left|\psi_{1}-\psi_{2}\right|\right]+} \\
& \frac{1}{H_{1} H_{2}}\left[\frac{C\left(1+\left\|f_{2}\right\|\right)}{\beta^{\frac{5}{2}}\left(s_{1}\right) \beta^{3}\left(z_{1}\right)}\right]\left|H_{2}-H_{1}\right| \\
& \left.\leq \frac{1}{H_{1} H_{2}}\left[\frac{C\left(1+\left\|f_{1}\right\|+\left\|f_{2}\right\|\right.}{\beta^{4}\left(s_{1}\right) \beta^{4}\left(s_{2}\right) \beta^{6}\left(z_{1}\right) \beta^{4}\left(z_{2}\right)}+1+m_{0}^{2} \mid \phi_{1}+\phi_{2}\right)\right]\left[\left|H_{2}-H_{1}\right|+\left|s_{1}-s_{2}\right|+\left|z_{1}-z_{2}\right|\right] \\
& +\frac{1}{H_{1} H_{2}}\left[\frac{C\left(1+\left\|f_{1}\right\|+\left\|f_{2}\right\|\right)}{\beta^{4}\left(s_{1}\right) \beta^{4}\left(s_{2}\right) \beta^{6}\left(z_{1}\right) \beta^{4}\left(z_{2}\right)}+1+m_{0}^{2}\left|\phi_{1}+\phi_{2}\right|\right]\left[|| f_{1}-f_{2} \|+\left|\phi_{1}-\phi_{2}\right|+\left|\psi_{1}-\psi_{2}\right|\right] \\
& \leq \frac{1}{H_{1} H_{2}}\left[\frac{C\left(1+\left\|f_{1}\right\|+\left\|f_{2}\right\|\right)}{\beta^{4}\left(s_{1}\right) \beta^{4}\left(s_{2}\right) \beta^{6}\left(z_{1}\right) \beta^{4}\left(z_{2}\right)}+1+m_{0}^{2}\left|\phi_{1}+\phi_{2}\right|\right]\left[\left|H_{2}-H_{1}\right|+\left|s_{1}-s_{2}\right|+\left|z_{1}-z_{2}\right|\right]+
\end{aligned}
$$




$$
\left.+\frac{1}{H_{1} H_{2}}\left[\frac{C\left(1+\left\|f_{1}\right\|+\left\|f_{2}\right\|\right)}{\beta^{4}\left(s_{1}\right) \beta^{4}\left(s_{2}\right) \beta^{6}\left(z_{1}\right) \beta^{4}\left(z_{2}\right)}+1+m_{0}^{2}\left|\phi_{1}+\phi_{2}\right|\right]||\left|f_{1}-f_{2} \|+\right| \phi_{1}-\phi_{2}|+| \psi_{1}-\psi_{2} \mid\right]
$$

this prove the proposition $7 .+$

Lemma 6: Let $\phi_{0}, \psi_{0} \in \mathrm{R}$ be given, then there exists the positive constants $M_{5}^{0}$ and $M_{4}^{0}$, such that

$$
\|\phi\| \leq M_{5}^{0},\|\psi\| \leq M_{4}^{0}
$$

where $M_{5}^{0}=M_{5}^{0}\left(H_{0}, T, \phi_{0}, \psi_{0}\right)$ and $M_{4}^{0}=M_{4}^{0}\left(H_{0}, T, \phi_{0}, \psi_{0}\right)$.

proof: Recall that $H \in] H_{1}^{0}, H_{0}\left[\right.$, we deduce from equations (38) and (39), that there exists $C=C\left(H_{0}\right)$ such that:

$$
|\dot{\phi}| \leq \sqrt{2\left(\psi_{0}+m_{0}^{2} T \max \left\{\phi_{0}^{2}, \phi^{2}(T)\right\}\right) e^{C T}}, t \in[0, \delta[
$$

Integration (93) over $[0, \delta[, \delta \in[0, T[$, we have:

$$
|\phi(t)| \leq\left|\phi_{0}\right|+(1+T) \sqrt{2\left(\psi_{0}+m_{0}^{2} T \max \left\{\phi_{0}^{2}, \phi^{2}(T)\right\}\right) e^{C T}}
$$

Finaly, we obtain $\|\psi\| \leq M_{4}^{0}$ and $\|\phi\| \leq M_{5}^{0}{ }^{+}$

Lemma 7: Let $\left.\left.\phi_{1}, \psi_{1}, \phi_{2}, \psi_{2} \in \mathrm{R}, H \in\right] 0, H_{0}\right]$, then

$$
\left|h\left(H, s, z, \Sigma_{+}, \phi_{1}, \psi_{1}, f, \bar{p}\right)-h\left(H, s, z, \Sigma_{+}, \phi_{2}, \psi_{2}, f, \bar{p}\right)\right| \leq N_{1}\left[\left|\psi_{1}-\psi_{2}\right|+\left|\phi_{1}-\phi_{2}\right|\right]
$$

Where

$$
N_{1}=6 H_{0}+\frac{m_{0}^{2} M_{5}^{0}+1}{\sqrt{\psi_{0}}}+m_{0}^{2} \sqrt{2 M_{4}^{0}}
$$

Proof: We have, taking account of equations (38) and (39)

$$
\begin{aligned}
& \left|h\left(H, s, z, \Sigma_{+}, \phi_{1}, \psi_{1}, f, \bar{p}\right)-h\left(H, s, z, \Sigma_{+}, \phi_{2}, \psi_{2}, f, \bar{p}\right)\right| \\
& \leq\left|\sqrt{2 \psi_{1}}-\sqrt{2 \psi_{2}}\right|+6 H\left|\psi_{1}-\psi_{2}\right|+m_{0}^{2} \sqrt{2 \psi_{1}}\left|\phi_{1}-\phi_{2}\right|+m_{0}^{2} \phi_{2}\left|\sqrt{2 \psi_{1}}-\sqrt{2 \psi_{2}}\right| \\
& \leq \frac{\left(2+2 m_{0}^{2}\left|\phi_{2}\right|\right) \psi_{1}-\psi_{2} \mid}{\sqrt{2 \psi_{1}}+\sqrt{2 \psi_{2}}}+6 H\left|\psi_{1}-\psi_{2}\right|+m_{0}^{2} \sqrt{2 \psi_{1}}\left|\phi_{1}-\phi_{2}\right|
\end{aligned}
$$

Using $\left.H \in] 0, H_{0}\right]$, (27) and (95), we are able to write

$$
\left|h\left(H, s, z, \Sigma_{+}, \phi_{1}, \psi_{1}, f, \bar{p}\right)-h\left(H, s, z, \Sigma_{+}, \phi_{2}, \psi_{2}, f, \bar{p}\right)\right| \leq N_{1}\left[\left|\psi_{1}-\psi_{2}\right|+\left|\phi_{1}-\phi_{2}\right|\right]+
$$


Lemma 8: Let $X_{1}$ and $X_{2} \in D \times \mathrm{R} \times \mathrm{R} \times H_{d}^{3}\left(\mathrm{R}^{3}\right) \times \mathrm{R}^{3}$, then

$$
\begin{gathered}
\left\|h\left(X_{1}\right)-h\left(X_{2}\right)\right\|_{E} \leq \\
N\left[\begin{array}{l}
\left|H_{1}-H_{2}\right|+\left|s_{1}-s_{2}\right|+\left|z_{1}-z_{2}\right|+\left|\Sigma_{+1}-\Sigma_{+2}\right| \\
+\left\|f_{1}-f_{2}||+\left|\phi_{1}-\phi_{2}\right|+\left|\psi_{1}-\psi_{2}\right|+\right\|\left|p_{1}-\bar{p}_{2}\right| \mid
\end{array}\right]
\end{gathered}
$$

Where

$$
\begin{gathered}
N=6 H_{0}+\frac{m_{0}^{2} M_{5}^{0}+1}{\sqrt{\psi_{0}}}+m_{0}^{2} \sqrt{2 M_{4}^{0}}+ \\
C\left(1+\frac{1}{H_{1} H_{2}}\right)\left(1+\frac{C\left[3+\left\|f_{1}\right\|+\left\|f_{2}\right\|+\left\|f_{1}\right\|^{2}+\left\|f_{2}\right\|^{2}\right]}{\beta^{4}\left(s_{1}\right) \beta^{4}\left(s_{2}\right) \beta^{6}\left(z_{1}\right) \beta^{4}\left(z_{2}\right)}+1+m_{0}^{2}\left|\phi_{1}+\phi_{2}\right|\right)
\end{gathered}
$$

Proof: Using the function $\mathrm{h}$, we obtain:

$$
\begin{aligned}
& \left\|h\left(H_{1}, s_{1}, z_{1}, \Sigma_{+1}, \phi_{1}, \psi_{1}, f_{1}, \bar{p}_{1}\right)-h\left(H_{2}, s_{2}, z_{2}, \Sigma_{+2}, \phi_{2}, \psi_{2}, f_{2}, \bar{p}_{2}\right)\right\|_{E} \\
& \leq\left\|Z\left(H_{1}, s_{1}, z_{1}, \Sigma_{+1}\right)-Z\left(H_{2}, s_{2}, z_{2}, \Sigma_{+2}\right)\right\| \\
& +\sum_{i=1}^{3}\left\|\frac{a b^{2} g^{i i}\left(s_{1}, z_{1}\right) \varphi_{i k} p_{1}^{k}}{p^{0}\left(s_{1}, z_{1}, \bar{p}_{1}\right)} \int f_{1} d \bar{p}-\frac{a b^{2} g^{i i}\left(s_{2}, z_{2}\right) \varphi_{i k} p_{2}^{k}}{p^{0}\left(s_{2}, z_{2}, \bar{p}_{2}\right)} \int f_{2} d \bar{p}\right\| \\
& +\|\left(\frac{1}{p^{0}\left(s_{1}, z_{1}, \bar{p}_{1}\right)} Q\left(f_{1}, f_{1}\right)\left(s_{1}, z_{1}, \bar{p}_{1}\right)-\frac{1}{p^{0}\left(s_{2}, z_{2}, \bar{p}_{2}\right)} Q\left(f_{2}, f_{2}\right)\left(s_{2}, z_{2}, \bar{p}_{2}\right) \|\right. \\
& +\frac{1}{3} \sum_{i=1}^{2}\left|P_{i}\left(s_{1}, z_{1}, f_{1}, \phi_{1}, \psi_{1}\right)-P_{i}\left(s_{2}, z_{2}, f_{2}, \phi_{2}, \psi_{2}\right)\right| \\
& +\left|\Sigma_{+1}-\Sigma_{+2}\right|\left|\frac{P_{1}\left(s_{2}, z_{2}, f_{2}, \phi_{2}, \psi_{2}\right)}{H_{2}}\right| \\
& +\frac{1}{3}\left|\frac{P_{1}\left(s_{1}, z_{1}, f_{1}, \phi_{1}, \psi_{1}\right)}{H_{1}}-\frac{P_{1}\left(s_{2}, z_{2}, f_{2}, \phi_{2}, \psi_{2}\right)}{H_{2}}\right|\left|\Sigma_{+1}-2\right| \\
& +\frac{1}{3}\left|\frac{P_{2}\left(s_{1}, z_{1}, f_{1}, \phi_{1}, \psi_{1}\right)}{H_{1}}-\frac{P_{2}\left(s_{2}, z_{2}, f_{2}, \phi_{2}, \psi_{2}\right)}{H_{2}}\right| \Sigma_{+1}+1 \mid \\
& +\left|\Sigma_{+1}-\Sigma_{+2}\right|\left|\frac{P_{2}\left(s_{2}, z_{2}, f_{2}, \phi_{2}, \psi_{2}\right)}{H_{2}}\right|+\left|h\left(H, s, z, \Sigma_{+}, \phi_{1}, \psi_{1}, f, \bar{p}\right)-h\left(H, s, z, \Sigma_{+}, \phi_{2}, \psi_{2}, f, \bar{p}\right)\right|
\end{aligned}
$$

Using the inequalities (79), (86), (87), (90), (91) and lemma 7. We obtain the result. +

Proposition 9: There exists a real number $\delta>0$ such that the differential system (S) defined by (80), with the 
initial datum $\mathrm{X}_{0}$ at $\mathrm{t}=0$ ( adequately fixed as above) has a unique solution $X$ defined over $[0, \delta]$ and satisfying $X(0)=X_{0}$.

Proof: Let $\left.\left(H^{0}, s^{0}, z^{0}, \Sigma_{+}^{0}\right) \in\right] H_{1}^{0}, H^{0}[\times] 0,1[\times] 0,1[\times]-1,1\left[, \bar{p}_{0} \in \mathrm{R}^{3}, \phi_{0}, \psi_{0} \in \mathrm{R}, f_{0} \in H_{d}^{3}\left(\mathrm{R}^{3}\right)\right.$. Consider H ${ }_{i}, \mathrm{~s}_{i}, z_{i}, i=1,2$ such that :

$$
\left.\left.\left.H_{i} \in\right] H_{1}^{0}, H^{0}\right], s_{i} \in\right] \frac{s^{0}}{2}, \frac{s^{0}+1}{2}\left[, z_{i} \in\right] \frac{z^{0}}{2}, \frac{z^{0}+1}{2}[
$$

Also consider $f_{1}, f_{2} \in B\left(f_{0}, 1\right)$ the unit ball in $H_{d}^{3}\left(\mathrm{R}^{3}\right)$, and $\bar{p}_{1}, \bar{p}_{2} \in \mathrm{R}^{3} ; \phi_{1}, \phi_{2}, \psi_{1}, \psi_{2} \in \mathrm{R}$. Then $\mathrm{H}_{i}, S_{i}, z_{i}$, and $\phi_{i}, \psi_{i}, i=1,2$ satisfy the following inequalities:

$$
\left\{\begin{array}{l}
\frac{1}{\beta\left(s_{i}\right)} \leq \frac{2}{\beta\left(s^{0}\right)}, \frac{1}{\beta\left(z_{i}\right)} \leq \frac{2}{\beta\left(z^{0}\right)}, \frac{1}{H_{i}} \leq \frac{1}{H_{1}^{0}} \\
\frac{\psi_{0}}{2} \leq \psi_{i} \leq M_{4}^{0}, \frac{1}{\sqrt{2 \psi_{i}}} \leq \frac{1}{\sqrt{\psi_{0}}}, \phi_{0} \leq \phi(t) \leq M_{5}^{0}
\end{array} \quad i=1,2\right.
$$

and we have $\left\|f_{i}\right\| \leq\left\|f_{0}\right\|+1, i=1,2$. Consequently, we have in (97), using (98),

$$
N \leq N^{\prime}\left(H^{0}, s^{0}, z^{0}, f^{0}, H_{0}, s_{0}, z_{0}, \Sigma_{+0}, \phi_{0}, \psi_{0}, f_{0}, \bar{p}_{0}, M_{4}^{0}, M_{5}^{0}\right)
$$

(96) and (99) show that the function $h$ is locally Lipschitzian in $X$, proposition 9 then follows from the standard theory on the first order differential systems. +

Theorem 3: Let $d>\frac{5}{2}, r>0, f_{0} \in H_{d, r}^{3}\left(0, T, \mathrm{R}^{3}\right), a_{0}, b_{0} \dot{a}_{0}, \dot{b}_{0}, E^{i}, \varphi_{i j}, \phi_{0} \in \mathrm{R}, \Lambda \geq 0$, satisfying (40), (41), (42), (43), (44) and $\bar{p}_{0} \in \mathrm{R}^{3}$ be given. Then there exists a real number $\delta>0$ such that system has a unique solution $(a, b, \phi, f)$ on $[0, \delta]$. This solution provides the solution $\left(a, b, F^{0 i}, F_{i j}, \phi, f\right)$ to system verifying at the initial datum $\left(a_{0}, b_{0}, E^{i}, \varphi_{i j}, \phi_{0}, f_{0}\right)$ and satisfies:

$$
\left\{\begin{array}{l}
f \in H_{d, r}^{3}\left(0, T, \mathrm{R}^{3}\right),\|f\|_{H_{d}^{3}\left(0, T, \mathrm{R}^{3}\right)} \leq\left\|f_{0}\right\|_{H_{d}^{3}\left(\mathrm{R}^{3}\right)} \\
F^{0 i}=\frac{a_{0} b_{0}^{2}}{a b^{2}} E^{i}, \quad F_{i j}=\varphi_{i j} \\
\left(a, b, F^{0 i}, F_{i j}, \phi, f\right)(0)=\left(a_{0}, b_{0}, E^{i}, \varphi_{i j}, \phi_{0}, f_{0}\right) \\
\dot{a}(0)=\dot{a}_{0}, \dot{b}(0)=\dot{b}_{0}>0
\end{array}\right.
$$

Proof: The system (33)-(34)-(35)-(37)-(38)-(39) is equivalent to system (S). If we take $\mathrm{s}_{0}, z_{0}, H_{0}, \Sigma_{+0}$ as defined in (101) and $\bar{p}_{0} \in \mathrm{R}^{3}$, we obtain by the proposition 9 the unique solution $\mathrm{X}$ of (S) on $[0, \delta]$ which verifies $X(0)=X_{0} . f$ is also the solution of the Boltzmann equation given by theorem 1. $s, z, \phi$ and $\psi$ being given by proposition 9 , we use formula (49) which gives $a$ and $b$ as functions of $s$ and $z$. Consequently, $(a, b)$ is also the unique solution to system (33)-(35) given by theorem 2 .

Adding (36), we clearly obtain that system (2)-(5) has a unique solution $\left(a, b, F^{0 i}, F_{i j}, \phi, f\right)$ on $[0, \delta]$. The inequality $\|f\|_{H_{d}^{3}\left(0, T, \mathrm{R}^{3}\right)} \leq\left\|f_{0}\right\|_{H_{d}^{3}\left(\mathrm{R}^{3}\right)}$ is prove in $[2]+$ 


\section{THE GLOBAL EXISTENCE}

We now show that the local solution to Einstein-Maxwell-Boltzmann-scalar field system (2)-(6), whose existence is proved in Theorem 3, is in fact for the case $\Lambda \geq 0$ a global solution. We will use the similar method as the one used in [1] and [6].

Denote by $[0, T[$ the maximal existence domain of the solution, denoted here by $(a, b, \phi, f)$ and given by theorem 3, of the system, with the initial datum as state in theorem 3 . We want to prove that $T=+\infty$.

○ If $T=+\infty$, then the problem of global existence is solved.

○ If not, $T<+\infty$. Let $t_{0} \in[0, T[$, we will show that there exists a strictly positive number $\delta>0$ independent of $\mathrm{t}_{0}$, such that the system on $\left[t_{o}, t_{o}+\delta\right]$, with the initial data $\left(\vec{a}\left(t_{0}\right), \vec{b}\left(t_{0}\right), \vec{\phi}\left(t_{0}\right), \vec{f}\left(t_{0}\right)\right) \quad$ at $t=t_{0}$ has a unique solution $(a, b, \phi, f)$ on $\left[t_{0}, t_{0}+\delta\right]$. Then, by taking to such that $0<T-t_{0}<\frac{\delta}{2}$, hence $T<t_{0}+\frac{\delta}{2}$,

we can extend the solution $(a, b, \phi, f)$, which contains strictly $[0, T[$, and this contradicts the maximality of $T$. In order to simplify the notations, it will be enough if we could look for a number $\delta$ such that $0<\delta<1$.

\section{The functional framework}

Proposition 10: Let $t_{0} \in 0, T\left[\right.$ and $0<\delta<1$. Then, any solution $\left(H, s, z, \Sigma_{+}, \phi, \psi, f, \bar{p}\right)$ for the initial value problem for the system (S) defined by (80) on $\left[t_{0}, t_{0}+\delta\right]$, with the initial data at $t=t_{0}$ :

$$
\left(H, s, z, \Sigma_{+}, \phi, \psi, f, \bar{p}\right)\left(t_{0}\right)=\left(\stackrel{\square}{H}\left(t_{0}\right), \stackrel{\square}{\square}\left(t_{0}\right), \underset{z}{z}\left(t_{0}\right), \stackrel{\square}{\Sigma_{+}}\left(t_{0}\right), \stackrel{\square}{\phi}\left(t_{0}\right), \stackrel{\square}{\psi}\left(t_{0}\right), \stackrel{\square}{f}\left(t_{0}\right), \stackrel{-}{p}\left(t_{0}\right)\right)
$$

where $\left(\stackrel{\square}{H}, s, z, \Sigma_{+}, \bar{\phi}, \psi, \square, \bar{f}, \bar{p}\right)$ denotes the solution in $[0, T[$, satisfies the inequalities:

$$
\left.\frac{1}{H\left(t_{0}+t\right)} \leq M_{0} ; \quad \frac{1}{\alpha\left(s\left(t_{0}+t\right)\right)} \leq M_{0} ; \quad \frac{1}{\alpha\left(z\left(t_{0}+t\right)\right)} \leq M_{0} ; \quad t \in 0, \delta\right]
$$

where:

$$
M_{0}=M_{0}\left(a_{0}, b_{0}, \dot{a}_{0}, \dot{b}_{0}, T\right)=\left(\frac{1}{H_{0}}+\frac{1}{s_{0}}+\frac{1}{z_{0}}\right) e^{10 H_{0}(T+1)}
$$

in which $H_{0}, s_{0}, z_{0}$ are defined in terms of $a_{0}, b_{0}, \dot{a}_{0}, \dot{b}_{0}$ by (47).

Proof: Consider the solution $\left(\breve{H}, \breve{s}, \breve{z}, \breve{\Sigma}_{+}\right)$of subsystem $\left(\mathrm{S}_{1}\right)-\left(S_{2}\right)-\left(S_{3}\right)-\left(S_{4}\right)$ of (S) on $[0, T[$ with the initial datum $\left(H_{0}, s_{0}, z_{0}, \Sigma_{+0}\right)$, defined in terms $a_{0}, b_{0}, \dot{a}_{0}, \dot{b}_{0}$; Applying proposition 4 , where we consider $\left(\tilde{H}, \tilde{s}, \tilde{z}, \tilde{\Sigma}_{+}\right)\left(t_{0}\right)=\left(\breve{H}, \breve{s}, \breve{z}, \breve{\Sigma}_{+}\right) \quad$ and $\quad\left(H, s, z, \Sigma_{+}\right)\left(t_{0}\right)=\left(\breve{H}, \breve{s}, \breve{z}, \breve{\Sigma}_{+}\right)\left(t_{0}\right)=\left(\tilde{H}, \tilde{s}, \tilde{z}, \tilde{\Sigma}_{+}\right)\left(t_{0}\right) \quad$ because $t_{0} \in 0, T[$, invoking (69)-(71), and $0<\delta<1$, we obtain the proposition 9. +

In what follows, $M_{0}$ is the absolute constant defined by (102). We deduce from (47), and the expressions of $a$, $b$ in (49) that:

$$
\frac{1}{s} \leq M_{0} ; \quad \frac{1}{z} \leq M_{0} ; \quad a^{2} \leq M_{0}^{2} ; \quad b^{2} \leq 2 M_{0}^{2}
$$


We then deduce from the definition (47) of $z$ and $s$, in terms of $a$ and $b$, using $a^{2} \geq a_{0}^{2} \geq \frac{9}{4}>2$, and the inequality for $H$ in (101):

$$
\frac{1}{M_{0}} \leq z \leq \frac{1}{1+2 M_{0}^{-2}} ; \quad \frac{1}{M_{0}} \leq s=\frac{1}{1+2 a^{2} b^{-2}} \leq \frac{1}{1+2 M_{0}^{-2}} ; \quad \frac{1}{M_{0}} \leq H \leq H_{0}
$$

On the basis of (101), (102), (103), we now introduce the following functions spaces, for $t_{0} \in 0, T[$ and $\delta>0$ : $t_{0} \in[0, T[, \delta>0$ :

$$
\begin{aligned}
& \left.E_{t_{0}}^{\delta}=\left\{s \in C\left(\left[\left(t_{0}, t_{0}+\delta\right)\right] ; \mathrm{R}\right) ; \frac{1}{M_{0}} \leq s \leq \frac{M_{0}^{2}}{1+M_{0}^{2}} ; \frac{1}{\beta\left(s\left(t_{0}+t\right)\right)} \leq M_{0} ; t \in 0, \delta\right]\right\} \\
& \left.F_{1, t_{0}}^{\delta}=\left\{H \in C\left(\left[\left(t_{0}, t_{0}+\delta\right)\right] ; \mathrm{R}\right) ; \frac{1}{M_{0}} \leq H\left(t_{0}+t\right) \leq H_{o} ; t \in 0, \delta\right]\right\} \\
& \left.F_{2, t_{0}}^{\delta}=\left\{\Sigma_{+} \in C\left(t_{0}, t_{0}+\delta\right) ;-1 \leq \Sigma_{+}\left(t_{0}+t\right) \leq 1 ; t \in 0, \delta\right]\right\}
\end{aligned}
$$

One verifies easily that $E_{t_{0}}^{\delta}, F_{1, t_{0}}^{\delta}$ and $F_{2, t_{0}}^{\delta}$ are complete metric subspaces of the Banack space denoted $C\left(t_{0}, t_{0}+\delta\right)$ , of the continuous (and hence bounded) functions on the line segment $\left[t_{0}, t_{0}+\delta\right]$, endowed with the norm:

$$
\|u\|_{\infty}=\sup _{\left.t \in t_{0}, t_{0}+\delta\right]}|u(t)| ; \quad u \in C\left(t_{0}, t_{0}+\delta\right)
$$

Lemma 9: Let $f_{0} \in H_{d, r}^{3}\left(0, T, \mathrm{R}^{3}\right)$, be given, then there exists a positive constant

$$
\begin{array}{r}
M_{0}^{3}=M_{0}^{3}\left(H_{o}, s_{o}, z_{o}, T,\left|E^{i}\right|,\left|\varphi_{i j}\right|, r, \bar{p}_{0}\right), \text { such that } \\
\|\bar{p}\| \leq M_{0}^{3},
\end{array}
$$

for any solution $X$ of (S) defined by (80) on $[0, \delta]$.

Proof: We have $p^{0} \geq a \mid p^{i}$, using (103), we deduce that there exists a positive constant $M_{6}^{0}=M_{6}^{0}\left(M_{0}, T\right)$ such that:

$$
\frac{\left|p^{k}\right|}{p^{0}(s, z, \bar{p})} \leq M_{6}^{0}, k=1,2,3 .
$$

Since $\left.f \in H_{d, r}^{3}\left(0, T, \mathrm{R}^{3}\right), H \in\right] 0, H_{0}\left[,\left|\Sigma_{+}\right|<1\right.$, we deduce for $\left(S_{8}\right)-\left(S_{9}\right)-\left(S_{10}\right)$ that there exists two positive constants $M_{2}^{0}=M_{2}^{0}\left(H_{0}\right), M_{3}^{0}=M_{3}^{0}\left(r, T,\left|E^{i}\right|,\left|\varphi_{i j}\right|, M_{0}, \bar{p}_{0}\right)$ such that

$$
\left|p^{i}\right| \leq M_{2}^{0}\left|p^{i}\right|+M_{3}^{0}, \quad i=1,2,3 .
$$

Integrating over $[0, t[, t \in[0, T[$, we obtain

$$
\left|\int_{0}^{t} \dot{p}^{i}(s) d s\right| \leq \int_{0}^{t}\left|\dot{p}^{i}(s)\right| d s \leq M_{2}^{0} \int_{0}^{t}\left|p^{i}(s)\right| d s+M_{3}^{0} T
$$


It follows that

$$
\left|p^{i}(t)\right| \leq\left(\left|p^{i}(0)+M_{3}^{0} T\right|\right)+M_{2}^{0} \int_{0}^{t}\left|p^{i}(s)\right| d s
$$

By the Gronwall lemma, we obtain

$$
\left|p^{i}(t)\right| \leq\left(\left|p^{i}(0)+M_{3}^{0} T\right|\right) e^{M_{2}^{0} T}=M_{0}^{3}, i=1,2,3 .
$$

Consequently, using (103) which gives $M_{0}=M_{0}\left(H_{0}, s_{0}, z_{0}, T\right)$, we obtain $\|\bar{p}\| \leq M_{0}^{3}+$

Global existence we set, for $i, j=1,2,3$

$$
\left\{\begin{array}{c}
\frac{d p^{i}}{d t}=-2 \Gamma_{0 j}^{i} p^{j}+\left(-a_{0} b_{0}^{2} E^{i}+\frac{a b^{2} p^{k} g^{i j} \varphi_{k j}}{p^{o}}\right) \int_{\mathrm{R}^{3}} f d \bar{p} \\
\frac{d f}{d t}=\frac{1}{p^{0}} Q(f, f, \bar{p}) \\
\frac{d \phi}{d t}=\sqrt{2 \psi} \\
\frac{d \psi}{d t}=-6 \Gamma_{0 j}^{i} \psi-m_{0}^{2} \phi \sqrt{2 \psi}-1
\end{array}\right.
$$

Lemma 10: Let $a, b$ be fixed, $t_{o} \geq 0,\left(\bar{p}_{t_{o}}, f_{t_{o}}, \phi_{t_{0}}, \psi_{t_{0}}\right) \in \mathrm{R}^{3} \times H_{d}^{3}\left(t_{0}, t_{0}+\delta ; \mathrm{R}^{3}\right) \times(\mathrm{R})^{2}$, then there exists $\delta>0$ such that system (107) has a unique local solution $\bar{p}, f, \phi, \psi) \in C\left(\left[\left(t_{0}, t_{0}+\delta\right)\right] ; \mathrm{R}^{3}\right) \times H_{d}^{3}\left(t_{0}, t_{0}+\delta ; \mathrm{R}^{3}\right) \times\left(ð\left(\left[t_{o}, t_{o}+\delta\right] ; \mathrm{R}\right)\right)^{2}$ and verifying at $t=t_{0}$ the relation ( $\bar{p}, f, \phi, \psi)\left(t_{0}\right)=\left(\bar{p}_{t_{o}}, f_{t_{o}}, \phi_{t_{0}}, \psi_{t_{0}}\right)$.

proof: We set $\stackrel{\square}{G}(t, \bar{p}, f, \phi, \psi)=\left(\vec{G}_{1}(t, \bar{p}, f), \breve{G}_{2}(t, \bar{p}, f), \stackrel{\square}{H}_{1}(t, \phi, \psi), \stackrel{H}{H}_{2}(t, \phi, \psi)\right)$, defined by the r.h.s of (107). Let $t_{0} \geq 0$ be an arbitrary real number. Since the functions $\gamma=a, b, a, b, \frac{1}{a}, \frac{1}{b}, B$, are continous of $t$, so is the function $\stackrel{\square}{G}$. By the continuity of $\gamma=a, b, \frac{1}{a}, \frac{1}{b}$ at $t=t_{0}$, there exists $\delta_{0}>0$ such that $\left.t \in\right] t_{0}-\delta_{0} ; t_{0}+\delta_{0}[$ implies that

$$
|\gamma(t)| \leq\left|\gamma\left(t_{0}\right)\right|+1
$$

Now, using the corollary 1 and remark 6, (108) then gives

$$
|\gamma(t)| \leq\left(a_{t_{0}}+b_{t_{0}}+\frac{1}{a_{t_{0}}}+\frac{1}{b_{t_{0}}}\right) e^{C t_{0}}+1
$$

Next, set

$$
B\left(f_{t_{0}}, 1\right)=\left\{f \in H_{d}^{3}\left(\mathrm{R}^{3}\right):\left\|f-f_{t_{0}}\right\|<1\right\},
$$

and consider the neighborhood $\left.W_{t_{0}}=\right] t_{0}-\delta_{0} ; t_{0}+\delta_{0}\left[\times \mathrm{R}^{3} \times B\left(f_{t_{0}}, 1\right) \times \mathrm{R}^{2}\right.$ of $\left(t_{0}, \bar{p}_{t_{0}}, f_{t_{0}}, \phi_{t_{0}}, \psi_{t_{0}}\right)$ in the space 
$\mathrm{R} \times \mathrm{R}^{3} \times H_{d}^{3}\left(t_{0}, t_{0}+\delta ; \mathrm{R}^{3}\right) \times \mathrm{R}^{2}$ and taking $\left(t, \bar{p}_{1}, f_{1}, \phi_{1}, \psi_{1}\right),\left(t, \bar{p}_{2}, f_{2}, \phi_{2}, \psi_{2}\right) \in W_{t_{0}}$. Using the scheme developed for the proof of lemma 5 , lemma 7 and proposition 6 , we have:

$$
\begin{aligned}
& \|\| \vec{G}_{1}\left(t, \bar{p}_{1}, f_{1}, \phi_{1}, \psi_{1}\right)-\stackrel{\square}{G}_{1}\left(t, \bar{p}_{2}, f_{2}, \phi_{2}, \psi_{2}\right) \| \leq M_{4}\left(\left\|\bar{p}_{1}-\bar{p}_{2}\right\|+\left\|f_{1}-f_{2}\right\|\right) \\
& \left\|\vec{G}_{2}\left(t, \bar{p}_{1}, f_{1}, \phi_{1}, \psi_{1}\right)-\bar{G}_{2}\left(t, \bar{p}_{2}, f_{2}, \phi_{2}, \psi_{2}\right)\right\| \leq M_{5}\left(\left\|\bar{p}_{1}-\bar{p}_{2}\right\|+\left\|f_{1}-f_{2}\right\|\right)
\end{aligned}
$$

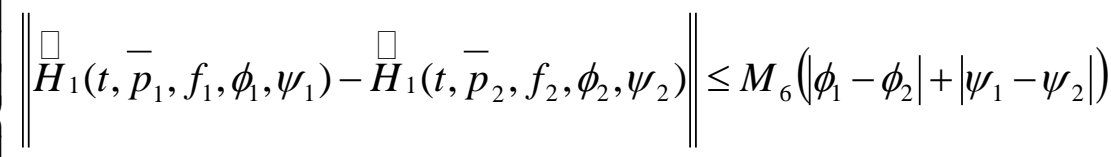

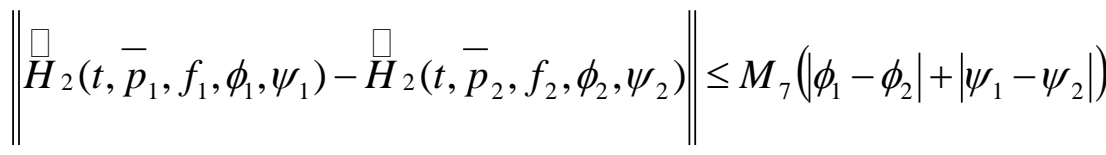

$$
\begin{aligned}
& \left\|\vec{G}\left(t, \bar{p}_{1}, f_{1}, \phi_{1}, \psi_{1}\right)-\stackrel{\square}{G}\left(t, \bar{p}_{2}, f_{2}, \phi_{2}, \psi_{2}\right)\right\| \leq \\
& M_{8}\left(|| \bar{p}_{1}-\bar{p}_{2}\|+\| f_{1}-f_{2} \|+\left|\phi_{1}-\phi_{2}\right|+\left|\psi_{1}-\psi_{2}\right|\right)
\end{aligned}
$$

where

$$
\left\{\begin{array}{c}
M_{4}=(C+1)\left(\frac{b^{2}}{a}+a\right)\left(1+\frac{a}{b}+\frac{b}{a}+\frac{1}{a}+\frac{1}{b}\right)\left(1+\left\|f_{2}\right\|+a_{t_{0}} b_{t_{0}}^{2}\left|E^{i}\right|++_{i}\left|\varphi_{i j}\right|\right. \\
M_{5}=8 \pi C_{1} a b^{2}(1+a+2 b)\left(1+\left\|f_{2}\right\|+\left\|f_{1}\right\|+\left\|f_{2}\right\|^{2}\right) ; M_{6}=\frac{1}{\sqrt{\psi_{t_{0}}}}, \\
M_{7}=2 H_{t_{0}}+\frac{m_{0}^{2}}{\sqrt{\psi_{t_{0}}}} M_{5}^{0}+m_{0}^{2} \sqrt{2 M_{4}^{0}} ; M_{8}=M_{4}+M_{5}+M_{6}+M_{7}
\end{array}\right.
$$

But by (109) and (112), we deduce from (111) that

$$
\left\|\vec{G}\left(t, \bar{p}_{1}, f_{1}, \phi_{1}, \psi_{1}\right)-\stackrel{\square}{G}\left(t, \bar{p}_{2}, f_{2}, \phi_{2}, \psi_{2}\right)\right\| \leq M_{8}^{\prime}\left(\left\|\bar{p}_{1}-\bar{p}_{2}\right\|+|| f_{1}-f_{2} \|+\left|\phi_{1}-\phi_{2}\right|+\left|\psi_{1}-\psi_{2}\right|\right)
$$

where

$$
M_{8}^{\prime}=M_{6}^{\prime}\left(a_{t_{0}}, b_{t_{0}}, t_{0},\left|E^{i}\right|,\left|\varphi_{i j}\right|, H_{t_{0}}, \psi_{t_{0}}, M_{4}^{0}, M_{5}^{0}, f_{t_{0}}\right) \geq M_{8}
$$

(113) and (114), show that $\vec{G}$ is lipschitzian in $(\bar{p}, f, \phi, \psi)$ with respect to the norm of the Banach space $\mathrm{R}^{3} \times H_{d}^{3}\left(\mathrm{R}^{3}\right) \times \mathrm{R}^{2}$. The existence of a unique solution $(\bar{p}, f, \phi, \psi)$ of system (107) on a interval $\left[t_{0}, t_{0}+\delta\right], \delta>0$ 
such that $(\bar{p}, f, \phi, \psi)\left(t_{0}\right)=\left(\bar{p}_{t_{0}}, f_{t_{0}}, \phi_{t_{0}}, \psi_{t_{0}}\right)$ is then guaranted by the standard theorem on the first order differential systems. +

proposition 11: Let $t_{0} \in[0, T[$, then there exists a real number $\delta>0$, depending only on the absolute constant $\mathrm{a}_{0}, b_{0}, \dot{a}_{0}, \dot{b}_{0}, T, \bar{p}_{0}, \phi_{0}, \psi_{0}$ and $r$ such that system (S) defined by (83) with the initial datum $\left(\stackrel{H}{H}\left(t_{0}\right), \vec{s}\left(t_{0}\right), z\left(t_{0}\right), \vec{\Sigma}_{+}\left(t_{0}\right), \vec{\phi}\left(t_{0}\right), \psi\left(t_{0}\right), \stackrel{\square}{f}\left(t_{0}\right), \bar{p}\left(t_{0}\right)\right)$ has a unique solution $\left(H, s, z, \Sigma_{+}, \phi, \psi, f, \bar{p}\right)$ belonging to $\left(E_{t_{0}}^{\delta}\right)^{2} \times F_{1, t_{0}}^{\delta} \times F_{2, t_{0}}^{\delta} \times\left(ð\left(\left[t_{0}, t_{0}+\delta\right] ; \mathrm{R}\right)\right)^{2} \times H_{d}^{3}\left(t_{0}, t_{0}+\delta ; \mathrm{R}^{3}\right) \times C\left(\left[\left(t_{0}, t_{0}+\delta\right)\right] ; \mathrm{R}^{3}\right)$.

Proof: By the above lemma 10, using Lemma 6 and Lemma 8 which show that $\mathrm{t} \mapsto \phi(t), t \mapsto \psi(t)$ and $\mathrm{t} \mapsto \bar{p}(t)$ are uniformly bounded, if we fix $(\bar{s}, \bar{z}) \in\left(E_{t_{0}}^{\delta}\right)^{2}$, and we define $\bar{a}=\bar{a}(\bar{s}, \bar{z}), \bar{b}=\bar{b}(\bar{s}, \bar{z})$ by (49). Then subsystem $\left(S_{5}\right)-\left(S_{6}\right) \quad-\quad\left(S_{7}\right)-\left(S_{8}\right)-\left(S_{9}\right)-\left(S_{10}\right) \quad$ of $\quad(\mathrm{S})$ has a unique solution $(\phi, \psi, \bar{p}, f) \in\left(ð\left(\left[t_{0}, t_{0}+\delta\right] ; \mathrm{R}\right)\right)^{2} \times C\left(\left[\left(t_{0}, t_{0}+\delta\right)\right] ; \mathrm{R}^{3}\right) \times H_{d}^{3}\left(t_{0}, t_{0}+\delta ; \mathrm{R}^{3}\right) \quad$ and $\quad$ verifying $(\phi, \psi, \bar{p}, f)\left(t_{0}\right)=\left(\square\left(t_{0}\right), \psi\left(t_{0}\right), \bar{p}_{t_{o}}, f_{t_{o}}\right)$.

Now if we substitute $\vec{f}$ to $f$ in (100) given by theorem 4, we obtain

$$
\|f(t)\|_{H_{d}^{3}\left(t_{0}, t_{0}+\delta ; \mathrm{R}^{3}\right)} \leq\left\|q_{t_{o}}\right\|_{H_{d}^{3}\left(\mathrm{R}^{3}\right)} \leq r, t \in\left[t_{o}, t_{o}+\delta\right]
$$

Next by proposition 5 there exists a real number $\delta$ belonging to $] 0,1\left[\right.$ such that if $\mathrm{f}$ is given in $H_{d, r}^{3}\left(t_{0}, t_{0}+\delta ; \mathrm{R}^{3}\right)$, then subsystem $\left(\mathrm{S}_{1}\right)-\left(S_{2}\right)-\left(S_{3}\right)-\left(S_{4}\right)$ of $(\mathrm{S})$ has a unique solution $\left(H, s, z, \Sigma_{+}\right)$on $\left[t_{0}, t_{0}+\delta\right]$ satisfying inequalities (78) and the condition $\left(H, s, z, \Sigma_{+}\right)\left(t_{0}\right)=\left(\stackrel{\square}{H}\left(t_{0}\right), \vec{\square}\left(t_{0}\right), \vec{z}\left(t_{0}\right), \square_{+}\left(t_{0}\right)\right)$.

Proposition 11 insures that $\left(H, s, z, \Sigma_{+}\right) \in\left(E_{t_{0}}^{\delta}\right)^{2} \times F_{1, t_{0}}^{\delta} \times F_{2, t_{0}}^{\delta}$.

Setting

$$
\left\{\begin{array}{c}
\Gamma_{t_{o}}^{\delta}=\left(E_{t_{0}}^{\delta}\right)^{2} \times H_{d, r}^{3}\left(t_{0}, t_{0}+\delta ; \mathrm{R}^{3}\right) \\
\gamma_{t_{0}}^{\delta}=F_{1, t_{0}}^{\delta} \times F_{2, t_{0}}^{\delta} \times \Gamma_{t_{o}}^{\delta} \times\left(ð\left(\left[t_{0}, t_{0}+\delta\right] ; \mathrm{R}\right)\right)^{2} \times \circlearrowright\left(\left[t_{0}, t_{0}+\delta\right] ; \mathrm{R}^{3}\right)
\end{array}\right.
$$

we can now define the map

$$
F: \Gamma_{t_{0}}^{\delta} \rightarrow \gamma_{t_{0}}^{\delta} ;(\bar{s}, \bar{z}, \bar{f}) \mapsto\left(H, \Sigma_{+}, s, z, f, \phi, \psi, \bar{p}\right)
$$

We are going to show that we can find a real number $\delta>0$ independent of $t_{0}$ such that, $\mathrm{F}$ defined above induces a contracting map of the complete metric space $\quad \Gamma_{t_{0}}^{\delta}$, which consequently will have a unique fixed point $(s, z, f)$. We will then deduce the existence of $H, \Sigma_{+}, \phi, \psi$ and $\bar{p}$ such that (S), admits the solution 
$\left(H, s, z, \Sigma_{+}, \phi, \psi, f, \bar{p}\right) \in \gamma_{t_{0}}^{\delta}$. If we fix in the r h s of $\left(S_{7}\right)-\left(S_{8}\right)-\left(S_{9}\right)-\left(S_{10}\right):(s=\bar{s}, z=\bar{z}) \in\left(E_{t_{0}}^{\delta}\right)^{2}$ and if we take in $\left(\mathrm{S}_{1}\right)-\left(S_{4}\right): P_{i}=\bar{P}_{i}=P_{i}(s, z, \bar{f}) ; i=1,2$; (where $(\bar{s}, \bar{z}) \in\left(E_{1, t_{0}}^{\delta} \times E_{2, t_{0}}^{\delta}\right)$ and $\bar{f} \in H_{d, r}^{3}\left(t_{0}, t_{0}+\delta ; \mathrm{R}^{3}\right)$; then the new system obtained, still called (S), admits the solution $\left(H, s, z, \Sigma_{+}, \phi, \psi, f, \bar{p}\right)$ belonging to $\gamma_{t_{0}}^{\delta}$ as indicated previously above and taking at $\mathrm{t}=\mathrm{t}_{0}$ initial datum $\left(\stackrel{\square}{H}\left(t_{0}\right), \vec{s}\left(t_{0}\right), \vec{z}\left(t_{0}\right), \stackrel{\square}{\Sigma_{+}}\left(t_{0}\right), \stackrel{\square}{\phi}\left(t_{0}\right), \underset{\psi}{\square}\left(t_{0}\right), \stackrel{\square}{f}\left(t_{0}\right), \bar{p}\left(t_{0}\right)\right)$. Moreover, $\left(H, s, z, \Sigma_{+}, \phi, \psi, f, \bar{p}\right)$ verifies the following integral system, with $\mathrm{t} \in[0, \delta], i=1,2,3$ :

$$
\begin{aligned}
H\left(t_{0}+t\right) & =\bar{H}\left(t_{0}\right)+\int_{t_{o}}^{t_{o}+t} Z_{1}\left(H, s, z, \Sigma_{+}, \phi, \psi, \bar{f}, \bar{p}\right)(\tau) d \tau \\
s\left(t_{0}+t\right) & =\bar{q}\left(t_{0}\right)+\int_{t_{o}}^{t_{o}+t} Z_{2}\left(H, s, z, \Sigma_{+}, \phi, \psi, f, \bar{p}\right)(\tau) d \tau \\
z\left(t_{0}+t\right) & =\bar{q}\left(t_{0}\right)+\int_{t_{o}}^{t_{o}+t} Z_{3}\left(H, s, z, \Sigma_{+}, \phi, \psi, f, \bar{p}\right)(\tau) d \tau \\
\Sigma_{+}\left(t_{0}+t\right) & =\bar{\Sigma}_{+}\left(t_{0}\right)+\int_{t_{o}}^{t_{o}+t} Z_{4}\left(H, s, z, \Sigma_{+}, \phi, \psi, \bar{f}, \bar{p}\right)(\tau) d \tau \\
\phi\left(t_{0}+t\right) & =\bar{\phi}\left(t_{0}\right)+\int_{t_{o}}^{t_{o}+t} h_{1}\left(H, s, z, \Sigma_{+}, \phi, \psi, f, \bar{p}\right)(\tau) d \tau \\
\psi\left(t_{0}+t\right) & =\psi\left(t_{0}\right)+\int_{t_{o}}^{t_{o}+t} h_{2}\left(H, s, z, \Sigma_{+}, \phi, \psi, f, \bar{p}\right)(\tau) d \tau \\
f\left(t_{0}+t\right) & =\bar{f}\left(t_{0}\right)+\int_{t_{o}}^{t_{o}+t} h_{3}\left(H, \bar{s}, \bar{z}, \Sigma_{+}, \phi, \psi, f, \bar{p}\right)(\tau) d \tau \\
& \square \\
p^{i}\left(t_{0}+t\right) & \bar{p}\left(t_{0}\right)+\int_{t_{o}}^{t_{o}+t} h_{4}\left(H, \bar{s}, \bar{z}, \Sigma_{+}, \phi, \psi, f, \bar{p}\right)(\tau) d \tau
\end{aligned}
$$

Let $\left(H_{1}, \Sigma_{+1}, s_{1}, z_{1}, f_{1}, \phi_{1}, \psi_{1}, \bar{p}_{1}\right)$ and $\left(H_{2}, \Sigma_{+2}, s_{2}, z_{2}, f_{2}, \phi_{2}, \psi_{2}, \bar{p}_{2}\right)$ be two solutions corresponding respectivily to $\left(\bar{s}_{1}, \bar{z}_{1}, \bar{f}_{1}\right)$ and $\left(\bar{s}_{2}, \bar{z}_{2}, \bar{f}_{2}\right)$ as obtained above. We write our integral system (118)-(125) for $\mathrm{i}=1,2$ next we take the difference. Taking now the devoted to local existence, we get

$$
\left\|f_{1}-f_{2}\right\| \leq \delta L_{1}^{0}\left(\left\|f_{1}-f_{2}\right\|+\left\|\bar{S}_{1}-\bar{s}_{2}\right\|_{\infty}+\left\|\bar{z}_{1}-\bar{z}_{2}\right\|_{\infty}+\|\| \bar{p}_{1}-\bar{p}_{2} \|\right)
$$

where $\quad L_{1}^{0}=C\left(1+2 r+2 r^{2}\right) M_{0}^{2}$

$$
\left\|\bar{p}_{1}-\bar{p}_{2}\right\| \leq \delta L_{2}^{0}\left(\left\|f_{1}-f_{2}\right\|+\left\|\bar{S}_{1}-\bar{s}_{2}\right\|_{\infty}+\left\|\bar{z}_{1}-\bar{z}_{2}\right\|_{\infty}+\left\|\bar{p}_{1}-\bar{p}_{2}\right\|\right)
$$

where $L_{2}^{0}=L_{2}^{0}\left(\left|E^{i}\right|,\left|\varphi_{i j}\right|, a_{0}, b_{0}, H_{0}, T, M_{0}, r\right)=C\left(1+a_{0} b_{0 i}^{2}\left|E^{i}\right|+H_{0}+M_{0}+r\right) M_{0}^{9}$.

$$
\left.\left\|H_{1}-H_{2}\right\|_{\infty} \leq \delta L_{3}^{o}\|\| \bar{f}_{1}-\bar{f}_{2}\|\|+\left\|H_{1}-H_{2}\right\|_{\infty}+\left\|s_{1}-s_{2}\right\|_{\infty}+\left\|z_{1}-z_{2}\right\|_{\infty}+\left\|\Sigma_{+1}-\Sigma_{+2}\right\|_{\infty}\right\rfloor
$$


where $L_{3}^{0}=L_{3}^{0}\left(a_{0}, b_{0}, \dot{a}_{0}, \dot{b}_{0}, r, T\right)=C\left(H_{0}\left(1+H_{0}\right)+(1+r) M_{0}^{16}\right.$

$$
\begin{gathered}
\left\|\Sigma_{+1}-\Sigma_{+2}\right\|_{\infty} \leq \\
\delta L_{4}^{o}\left\lfloor\left\|\bar{f}_{1}-\bar{f}_{2}\right\|+\left\|H_{1}-H_{2}\right\|_{\infty}+\left\|S_{1}-s_{2}\right\|_{\infty}+\left\|z_{1}-z_{2}\right\|_{\infty}+\left\|\Sigma_{+1}-\Sigma_{+2}\right\|_{\infty}\right]
\end{gathered}
$$

where $L_{4}^{0}=L_{4}^{0}\left(a_{0}, b_{0}, \dot{a}_{0}, \dot{b_{0}}, r, T\right)$

$$
\left.\left\|s_{1}-s_{2}\right\|_{\infty} \leq \delta L_{5}^{o}\left\|H_{1}-H_{2}\right\|_{\infty}+\left\|s_{1}-s_{2}\right\|_{\infty}+\left\|z_{1}-z_{2}\right\|_{\infty}+\left\|\Sigma_{+1}-\Sigma_{+2}\right\|_{\infty}\right]
$$

Where $L_{5}^{0}=L_{5}^{0}\left(a_{0}, b_{0}, \dot{a}_{0}, \dot{b}_{0}, r, T\right)$

$$
\left\|z_{1}-z_{2}\right\|_{\infty} \leq \delta L_{6}^{o}\left[\left\|H_{1}-H_{2}\right\|_{\infty}+\left\|s_{1}-s_{2}\right\|_{\infty}+\left\|z_{1}-z_{2}\right\|_{\infty}+\left\|\Sigma_{+1}-\Sigma_{+2}\right\|_{\infty}\right]
$$

where $L_{6}^{0}=L_{6}^{0}\left(a_{0}, b_{0}, a_{0}, b_{0}, r, T\right)$

$$
\left\|\phi_{1}-\phi_{2}\right\|_{\infty} \leq \delta L_{7}^{0}\left\|\psi_{1}-\psi_{2}\right\|_{\infty}
$$

where $L_{7}^{0}=\frac{1}{\psi_{0}}$

$$
\left\|\psi_{1}-\psi_{2}\right\|_{\infty} \leq \delta L_{8}^{0}\left(\left\|\psi_{1}-\psi_{2}\right\|_{\infty}+\left\|H_{1}-H_{2}\right\|_{\infty}+\left\|\phi_{1}-\phi_{2}\right\|_{\infty}\right)
$$

where $L_{8}^{0}=L_{8}^{0}\left(H_{0}, \psi_{0}, \phi_{0}, T\right)$

Summing inequalities (126)-(133) and taking first $\delta>0$ such that

$$
\delta\left(L_{1}^{0}+L_{2}^{0}+L_{3}^{o}+L_{4}^{o}+L_{5}^{o}+L_{6}^{o}+L_{7}^{0}+L_{8}^{0}\right)<\frac{1}{4}
$$

Then simplifying, we obtain

$$
\begin{aligned}
& \left\|H_{1}-H_{2}\right\|_{\infty}+\left\|s_{1}-s_{2}\right\|_{\infty}+\left\|z_{1}-z_{2}\right\|_{\infty}+\left\|\Sigma_{+1}-\Sigma_{+2}\right\|_{\infty}+\left\|\phi_{1}-\phi_{2}\right\|_{\infty} \\
& +\left\|\psi_{1}-\psi_{2}\right\|_{\infty}+\left\|f_{1}-f_{2}\right\|+\left\|\bar{p}_{1}-\bar{p}_{2}\right\| \leq \\
& \left.2 \delta\left(L_{1}^{0}+L_{2}^{0}+L_{3}^{o}+L_{4}^{o}\right)\left\|\bar{f}_{1}-\bar{f}_{2}\right\|+\left\|\bar{s}_{1}-\bar{s}_{2}\right\|_{\infty}+\left\|\bar{z}_{1}-\bar{z}_{2}\right\|_{\infty}\right) \leq \\
& \frac{1}{2}\left(\left\|\bar{f}_{1}-\bar{f}_{2}\right\|+\left\|\bar{s}_{1}-\bar{s}_{2}\right\|_{\infty}+\left\|\bar{z}_{1}-\bar{z}_{2}\right\|_{\infty}\right)
\end{aligned}
$$

because $2 \delta\left(L_{1}^{0}+L_{2}^{0}+L_{3}^{o}+L_{4}^{o}\right) \leq 2 \delta\left(L_{1}^{0}+L_{2}^{0}+L_{3}^{o}+L_{4}^{o}+L_{5}^{o}+L_{6}^{o}+L_{7}^{0}+L_{8}^{0}\right)<\frac{1}{2}$.

From which we deduce

$$
\left\|f_{1}-f_{2}\right\|+\left\|s_{1}-s_{2}\right\|_{\infty}+\left\|z_{1}-z_{2}\right\|_{\infty} \leq \frac{1}{2}\left[\left\|\bar{f}_{1}-\bar{f}_{2}\right\|\|+\| \bar{s}_{1}-\bar{s}_{2}\left\|_{\infty}+\right\| \bar{z}_{1}-\bar{z}_{2} \|_{\infty}\right]
$$

Consequently, if we take

$$
0<\delta<\inf \left\{1, \frac{1}{4\left(L_{1}^{0}+L_{2}^{0}+L_{3}^{o}+L_{4}^{o}+L_{5}^{o}+L_{6}^{o}+L_{7}^{0}+L_{8}^{0}\right)}\right\}
$$


Then inequality (136) insures that the map $F: \Gamma_{t_{0}}^{\delta} \rightarrow \gamma_{t_{0}}^{\delta} ;((\bar{s}, \bar{z}), \bar{f}) \mapsto\left(H, \Sigma_{+}, s, z, f, \phi, \psi, \bar{p}\right)$ defined by (117) induces a contraction $(\bar{s}, \bar{z}, \bar{f}) \mapsto(s, z, f)$ in the complete metric space $\Gamma_{t_{0}}^{\delta}$ for any real number $\delta$ satisfying (137). This shows that $\delta$ depends only on absolute constants $a_{o}, b_{o}, \dot{a}_{o}, \dot{b}_{o}, \phi_{0}, \psi_{0}$,

$$
f_{0},\left|E^{i}\right|,\left|\varphi_{i j}\right|, T, r, F \text { then has a unique fixed point }(s, z, f) \in \Gamma_{t_{0}}^{\delta} \text { solution of integral system (117)-(124) }
$$

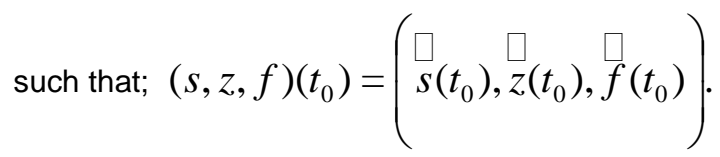

Now to determine $H, \Sigma_{+}, \bar{p}, \phi$ and $\psi$, consider system (S) in which we substitute $\bar{f}$ by $f, \vec{s}$ and $\square$ by $s$ and $z$. Since s is known, relation (57) determines the product $\Sigma_{+} H \quad$ as function of s. Since s and $\Sigma_{+} H$ are known, (58) provides $\mathrm{H}$ and (57) gives $\Sigma_{+}$. It remains to determine $\bar{p}$, from the three equations (S.8)-(S.9)-(S.10) of system (S) , Where $H, \Sigma_{+}, s, z$ and $f$ are known, we use the fact that,

$$
\left|\frac{p_{1}^{k}}{p_{1}^{0}}-\frac{p_{2}^{k}}{p_{2}^{0}}\right| \leq C M_{0}\left\|\bar{p}_{1}-\bar{p}_{2}\right\| \text { where } M_{0} \text { is given by (103), } \mathrm{C}>0 \text { is a constant. Since } H, \Sigma_{+} \text {are bounded, }
$$

the above inequality shows that subsystem (S.8)-(S.9)-(S.10) of system (S) for the single unknown $\bar{p}$ is globally lipschitzian in $\bar{p}$ : hence, there exists a unique solution $\bar{p}=\left(p^{i}\right)$ such that $\bar{p}\left(t_{0}\right)=\stackrel{\square}{p}\left(t_{0}\right)$, global on $\left[t_{0}, t_{0}+\delta\right]$. To determine $\phi$ and $\psi$, consider the equations $\left(S_{5}\right)-\left(S_{6}\right)$ of system (S), where $H$ is known and bounded. using (100) , the subsystem $\left(S_{5}\right)-\left(S_{6}\right)$ of system (S) is globally lipschitzian in $\phi$ and $\psi$ : hence there exists a unique solution $(\phi, \psi)$ such that $(\phi, \psi)\left(t_{0}\right)=\left(\square\left(t_{0}\right), \psi\left(t_{0}\right)\right)$ globale sur $\left[t_{0}, t_{0}+\delta\right]$

Consequently, we obtain the unique solution $\left(H, s, z, \Sigma_{+}, \phi, \psi, f, \bar{p}\right)$ of system (S) in $\gamma_{t_{0}}^{\delta}+$

Theorem4:Let

$$
\phi_{0}, \psi_{0} \in \mathrm{R}, \Lambda \geq 0, r>0, d>\frac{5}{2}, a_{0}, b_{0}, \dot{a}_{0}
$$

$\dot{b}_{0} \in \mathrm{R}, \bar{p}_{0} \in \mathrm{R}^{3}, f_{0} \in H_{d, r}^{3}\left(\mathrm{R}^{3}\right), F^{0 i}(0)=E^{i} \in \mathrm{R}, F_{i j}(0)=\varphi_{i j} \in \mathrm{R}$

such that $a_{0}, b_{0}, \dot{a}_{0}, \dot{b}_{0}, \phi_{0}, \psi_{0}, f_{0}, E^{i}, \varphi_{i j}$ verify the constraints (40), (42), (43) and (44). Then:

1- differential system $\left(\mathrm{S}_{1}\right)-\left(S_{2}\right)-\left(S_{3}\right)-\left(S_{4}\right)-\left(S_{5}\right)-\left(S_{6}\right)-\left(S_{7}\right)-\left(S_{8}\right)-\left(S_{9}\right)-\left(S_{10}\right)$ has a unique global solution $\left(H, s, z, \Sigma_{+}, \phi, \psi, f, \bar{p}\right)$ defined on $[0,+\infty[$ and verifying $\left(H, s, z, \Sigma_{+}, \phi, \psi, f, \bar{p}\right)(0)=\left(H_{0}, s_{0}, z_{0}, \Sigma_{+0}, \phi_{0}, \psi_{0}, f_{0}, \bar{p}_{0}\right)$.

2- the coupled system, in a locally rotationally symmetric Bianchi type 1 space-time, has a unique global regular solution $\left(a, b, F^{0 i}, F_{i j}, f, \phi\right) \quad$ defined on $\quad[0,+\infty[$ and verifying $a(0)=a_{0}, b(0)=b_{0}, F^{0 i}=\frac{a_{0} b_{0}^{2}}{a b^{2}} E^{i}, F^{0 i}(0)=E^{i}, F_{i j}(0)=\varphi_{i j}, f(0)=f_{0}$ and $\phi(0)=\phi_{0}$. 


\section{REFERENCES}

[1] Noutchegueme, N. and Dongo, D. 2005. global existence of solutions for the Einstein-Boltzmann system in a Bianchi type 1 space-time for arbitrarily large initial data, classical quantum gravity, vol $23, n^{\circ} 9$, 2979-3003.

[2] Noutchegueme, N. and Kenmogne, M. N. 2015. Global regular solution to Boltzmann equation a Roberson Walker, International journal of physics and Mathematical Sciences ISSN, vol 5, 2277-2111.

[3] Noutchegueme, N., Dongo, D., and Takou, E. 2005. global existence of solutions for the relativistic Boltzmann equation with arbitrarily large initial data on a Bianchi type 1 space-time, Gen.Rel.Grav 2047-2062.

[4] Noutchegueme, N. and Ayissi, R. D. 2010. global existence of solution to the Maxwell-Boltzmann system in a Bianchi type 1 space-time, Advanced studies in theoretical physic, vol $4, \mathrm{n}^{\circ}$ 17-20, 855-878.

[5] Ayissi, R. D. and Noutchegueme, N. 2013. Global regular solutions to Maxwell-Boltzmann-Euler system a Bianchi type 1 space-time in presence of a massive scalar field, International journal of research and reviews in applied sciences, vol 14, $\mathrm{n}^{\circ} 2,276-315$ (2013).

[6] Noutchegueme, N. and Ayissi, R. D. 2015. Bianchi type 1 magnetized cosmological models for the Einstein-Boltzmann equation with the cosmological constant, journal of mathematical physics, vol 56 .

[7] Jantzen, R. T. 1984.Cosmology of the Early Universe ed L Z Fang and R Ruffini(Singapore: World Scientific).

\section{Author biography with photo}

1 st Author: NOUTCHEGUEME Norbert Birth 1950 ; 1975-1981 Assistant; 1981-1988 :Lecturer; 1988-2001: Associate Professor From 2001:Full Professor. He has supervised 14 Doctorate Theses. He has 52 publications. 4 Students became Associate Professors.

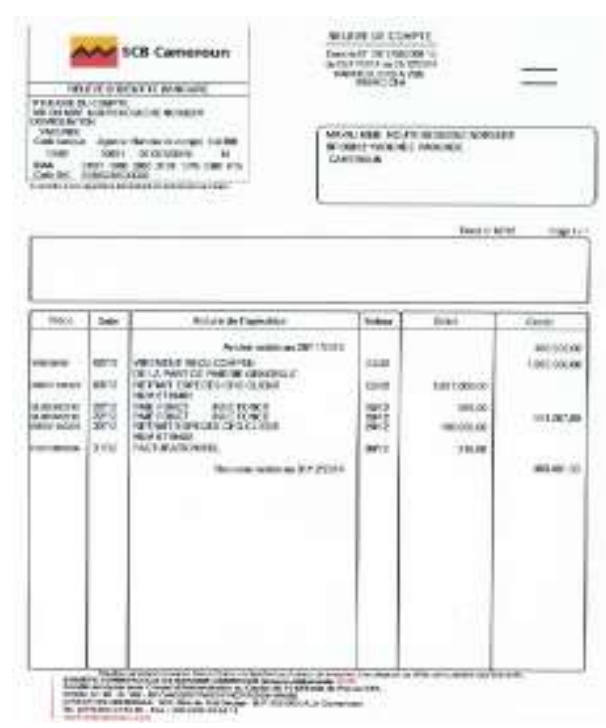

2nd Author: DONGO David Birth 1975; Assitant lecturer: 2005-2006; Lecturer from 2006. He has 3 publications.

3rd Author: DJIOFACK Francis Etienne birth 1987; Student in doctorat/phd from $2014 .$. 